\title{
CONSTRUCTION OF CONSTANT MEAN CURVATURE $n$-NOIDS USING THE DPW METHOD
}

\author{
MARTIN TRAIZET
}

\begin{abstract}
: we construct constant mean curvature surfaces in euclidean space with genus zero and $n$ ends asymptotic to Delaunay surfaces using the DPW method.
\end{abstract}

\section{INTRODUCTION}

In [3], Dorfmeister, Pedit and $\mathrm{Wu}$ have shown that harmonic maps from a Riemann surface to a symmetric space admit a Weierstrass-type representation, which means that they can be represented in terms of holomorphic data. In particular, surfaces with constant mean curvature one (CMC-1 for short) in euclidean space admit such a representation, owing to the fact that the Gauss map of a CMC-1 surface is a harmonic map to the 2 -sphere. This representation is now called the DPW method and has been widely used to construct CMC- 1 surfaces in $\mathbb{R}^{3}$ and also constant mean curvature surfaces in homogeneous spaces such as the sphere $\mathbb{S}^{3}$ or hyperbolic space $\mathbb{H}^{3}$ : see for example [2, 4, 10, 11, 12, 13, 15, 16, 21, 22. . Also the DPW method has been implemented by N. Schmitt to make computer images of CMC-1 surfaces.

The main limitation to the construction of examples is the Monodromy Problem, so either the topology of the constructed examples is limited or symmetries are imposed to the construction, in order to reduce the number of equations to be solved.

In constract, Kapouleas [14] has constructed embedded CMC-1 surfaces with no limitation on the genus or number of ends by gluing round spheres and pieces of Delaunay surfaces, using Partial Differential Equations techniques. An interesting question is wether similar results can be achieved with the DPW method. In this paper, we make a first step in this direction by constructing $n$-noids: genus zero CMC- 1 surfaces with $n$ ends.

Theorem 1. Given $n \geq 3$ distinct unit vectors $u_{1}, \cdots, u_{n}$ in $\mathbb{R}^{3}$ and $n$ non-zero real weights $\tau_{1}, \cdots, \tau_{n}$ satisfying the balancing condition

$$
\sum_{i=1}^{n} \tau_{i} u_{i}=0
$$

there exists a smooth 1-parameter family of CMC-1 surfaces $\left(M_{t}\right)_{0<t<\varepsilon}$ with genus zero, $n$ Delaunay ends and the following properties:

1. If we denote $w_{i, t}$ the weight of the $i$-th Delaunay end and $\Delta_{i, t}$ its axis, then

$$
\lim _{t \rightarrow 0} \frac{w_{i, t}}{t}=8 \pi \tau_{i}
$$

and $\Delta_{i, t}$ converges to the half-line through the origin directed by $u_{i}$.

2. If all weights $\tau_{i}$ are positive, then $M_{t}$ is Alexandrov-embedded.

3. If moreover the angle between $u_{i}$ and $u_{j}$ is greater than $\frac{\pi}{3}$ for all $j \neq i$, then $M_{t}$ is embedded.

These examples can be described heuristically as the unit sphere with $n$ half Delaunay surfaces with small necksizes attached at the points $u_{1}, \cdots, u_{n}$ (see Figure 1). They are a particular case of the construction of Kapouleas [14]. Here are some related results: 


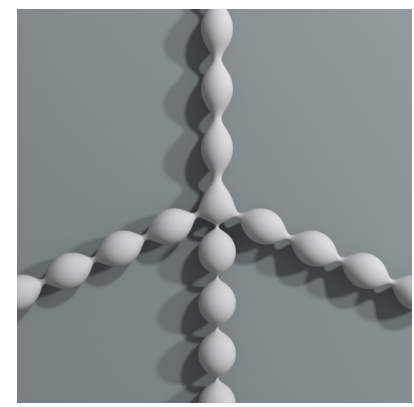

FiguRE 1. a symmetric 4-noid. Image by N. Schmitt

1. The full family of 3-noids is constructed in [4, 22] using the DPW method. Their construction is more general than ours in the case $n=3$, since we only construct 3 -noids whose ends have small necksizes.

2. Highly symmetric $n$-noids with $n \geq 4$ have been constructed using the DPW method in [16, 21].

3. DPW potentials of general $n$-noids are investigated in [4, 7], but the Monodromy Problem is not solved when $n \geq 4$. Quoting [7]: Outside of experimental examples, the construction of $k$-noids for $k \geq 4$, even under the additional assumption of coplanarity, remains elusive from the integrable systems perspective.

4. Alexandrov-embedded 3-noids have been classified in [8], and Alexandrov-embedded $n$-noids with coplanar ends have been classified in [9] (with methods unrelated to DPW).

5. The balancing condition of Theorem 1 is necessary by the general balancing formula for CMC-1 surfaces (Theorem 3.4 in [18]).

Our goal in this paper is to prove Theorem 1 with the DPW method, using a rather simple and natural DPW potential, inspired from the one used in [22] for 3-noids. Our main motivation is to make progress in the DPW method. In this regard, here is what is achieved in this paper:

1. We are able to solve the Monodromy Problem on an $n$-punctured sphere, whose fundamental group has $n-1$ generators, without any symmetry assumption. The Monodromy Problem is solved by an Implicit Function argument in a suitable functional space.

2. We are able to prove embeddedness. I believe this is the first time that a non-trivial example is proven to be embedded using the DPW method. The proof relies on the study of Delaunay ends in [17] and [20.

3. The DPW potential that we use has the property that its poles depend on the spectral parameter $\lambda$. This problem is answered by Theorem 3 in Section 3 , a general result which allows $\lambda$-dependent changes of variable in the DPW method. Theorem 3 adds some flexibility to the choice of the DPW potential.

\section{BACKGROUND}

In this section, we recall standard notations and results used in the DPW method. We work in the "untwisted" setting. For a comprehensive introduction to the DPW method, we suggest [6].

2.1. Loop groups. A loop is a smooth map from the unit circle $\mathbb{S}^{1}=\{\lambda \in \mathbb{C}:|\lambda|=1\}$ to a matrix group. The circle variable is denoted $\lambda$ and called the spectral parameter. For $\rho>0$, we denote $\mathbb{D}_{\rho}=\{\lambda \in \mathbb{C}:|\lambda|<\rho\}, \mathbb{D}_{\rho}^{*}=\mathbb{D}_{\rho} \backslash\{0\}$ and $\mathbb{D}=\mathbb{D}_{1}$.

- If $G$ is a matrix Lie group (or Lie algebra), $\Lambda G$ denotes the group (or algebra) of smooth maps $\Phi: \mathbb{S}^{1} \rightarrow G$. 
- $\Lambda_{+} S L(2, \mathbb{C}) \subset \Lambda S L(2, \mathbb{C})$ is the subgroup of smooth maps $B: \mathbb{S}^{1} \rightarrow S L(2, \mathbb{C})$ which extend holomorphically to $\mathbb{D}$.

- $\Lambda_{+}^{\mathbb{R}} S L(2, \mathbb{C})$ is the subgroup of $B \in \Lambda_{+} S L(2, \mathbb{C})$ such that $B(0)$ is upper triangular with positive elements on the diagonal.

Theorem 2 (Iwasawa decomposition). The multiplication $\Lambda S U(2) \times \Lambda_{+}^{\mathbb{R}} S L(2, \mathbb{C}) \rightarrow \Lambda S L(2, \mathbb{C})$ is a diffeomorphism. The unique splitting of an element $\Phi \in \Lambda S L(2, \mathbb{C})$ as $\Phi=F B$ with $F \in \Lambda S U(2)$ and $B \in \Lambda_{+}^{\mathbb{R}} S L(2, \mathbb{C})$ is called Iwasawa decomposition. $F$ is called the unitary factor of $\Phi$ and denoted Uni $(\Phi)$. $B$ is called the positive factor and denoted $\operatorname{Pos}(\Phi)$.

2.2. The matrix model of $\mathbb{R}^{3}$. In the DPW method, one identifies $\mathbb{R}^{3}$ with the Lie algebra $\mathfrak{s u}(2)$ by

$$
x=\left(x_{1}, x_{2}, x_{3}\right) \in \mathbb{R}^{3} \longleftrightarrow X=\frac{-\mathrm{i}}{2}\left(\begin{array}{cc}
-x_{3} & x_{1}+\mathrm{i} x_{2} \\
x_{1}-\mathrm{i} x_{2} & x_{3}
\end{array}\right) \in \mathfrak{s u}(2) .
$$

(This is essentially the same as identifying $\mathbb{R}^{3}$ with imaginary quaternions and using a matrix model for quaternions.) Under this identification, the euclidean scalar product and norm are given by

$$
\langle x, y\rangle=-2 \operatorname{tr}(X Y), \quad\|x\|^{2}=4 \operatorname{det}(X) .
$$

The group $S U(2)$ acts as linear isometries on $\mathfrak{s u}(2)$ by $H \cdot X=H X H^{-1}$.

2.3. The DPW method. The input data for the DPW method is a quadruple $\left(\Sigma, \xi, z_{0}, \phi_{0}\right)$ where:

- $\Sigma$ is a Riemann surface.

- $\xi=\xi(z, \lambda)$ is a $\Lambda \mathfrak{s l}(2, \mathbb{C})$-valued holomorphic 1 -form on $\Sigma$ called the DPW potential. More precisely,

$$
\xi=\left(\begin{array}{cc}
\alpha & \lambda^{-1} \beta \\
\gamma & -\alpha
\end{array}\right)
$$

where $\alpha(z, \lambda), \beta(z, \lambda), \gamma(z, \lambda)$ are holomorphic 1 -forms on $\Sigma$ with respect to the $z$ variable, and are holomorphic with respect to $\lambda$ in the disk $\mathbb{D}_{\rho}$ for some $\rho>1$.

- $z_{0} \in \Sigma$ is a base point.

- $\phi_{0} \in \Lambda S L(2, \mathbb{C})$ is an initial condition.

Given this data, the DPW method is the following procedure. Let $\widetilde{\Sigma}$ be the universal cover of $\Sigma$ and $\widetilde{z}_{0} \in \widetilde{\Sigma}$ be an arbitrary element in the fiber of $z_{0}$.

1. Solve the Cauchy Problem on $\widetilde{\Sigma}$ :

$$
d_{z} \Phi(z, \lambda)=\Phi(z, \lambda) \xi(z, \lambda)
$$

with initial condition

$$
\Phi\left(\widetilde{z}_{0}, \lambda\right)=\phi_{0}(\lambda)
$$

to obtain a solution $\Phi: \widetilde{\Sigma} \rightarrow \Lambda S L(2, \mathbb{C})$. (The notation $d_{z}$ means that we are considering the differential with respect to the $z$-variable. The lift of $\xi$ to $\widetilde{\Sigma}$ is still denoted $\xi$.)

2. Compute, for $z \in \widetilde{\Sigma}$, the unitary part $F(z, \cdot)=\operatorname{Uni}(\Phi(z, \cdot))$ in the Iwasawa decomposition of $\Phi(z, \cdot)$. It is known that $F(z, \lambda)$ depends real-analytically on $z$.

3. Define $f: \widetilde{\Sigma} \rightarrow \mathfrak{s u}(2) \sim \mathbb{R}^{3}$ by the Sym-Bobenko formula:

$$
f(z)=\mathrm{i} \frac{\partial F}{\partial \lambda}(z, 1) F(z, 1)^{-1}=: \operatorname{Sym}(F(z, \cdot)) .
$$

Then $f$ is a CMC-1 (branched) conformal immersion. Its Gauss map is given by

$$
N(z)=\frac{-\mathrm{i}}{2} F(z, 1)\left(\begin{array}{cc}
1 & 0 \\
0 & -1
\end{array}\right) F(z, 1)^{-1}=: \operatorname{Nor}(F(z, \cdot)) .
$$

Moreover, $f$ is regular at $z$ (meaning unbranched) if and only if $\beta(z, 0) \neq 0$. 
Observe that we require the potential $\xi$ to be holomorphic on $\Sigma$. In many examples, $\Sigma$ is a compact Riemann surface $\bar{\Sigma}$ minus a finite number of points, and $\xi$ extends meromorphically at the punctures.

2.4. The Monodromy Problem. Assume that $\Sigma$ is not simply connected so its universal cover $\tilde{\Sigma}$ is not trivial. Let $\operatorname{Deck}(\widetilde{\Sigma} / \Sigma)$ be the group of fiber-preserving diffeomorphisms of $\widetilde{\Sigma}$. For $\gamma \in \operatorname{Deck}(\widetilde{\Sigma} / \Sigma)$, let

$$
\mathcal{M}_{\gamma}(\Phi)(\lambda)=\Phi(\gamma(z), \lambda) \Phi(z, \lambda)^{-1}
$$

be the monodromy of $\Phi$ with respect to $\gamma$ (which is independent of $z \in \widetilde{\Sigma}$ ). The standard condition which ensures that the immersion $f$ descends to a well defined immersion on $\Sigma$ is the following system of equations, called the Monodromy Problem.

$$
\forall \gamma \in \operatorname{Deck}(\widetilde{\Sigma} / \Sigma) \quad \begin{cases}\mathcal{M}_{\gamma}(\Phi) \in \Lambda S U(2) & (i) \\ \mathcal{M}_{\gamma}(\Phi)(1)= \pm I_{2} & (i i) \\ \frac{\partial \mathcal{M}_{\gamma}(\Phi)}{\partial \lambda}(1)=0 & (\text { iii })\end{cases}
$$

Indeed, Condition (i) implies that $F$ has a monodromy, equal to the monodromy of $\Phi$, and provided this is true, Conditions (ii) and (iii) are equivalent to the fact that $f$ is well defined on $\Sigma$.

One can identify $\operatorname{Deck}(\widetilde{\Sigma} / \Sigma)$ with the fundamental group $\pi_{1}\left(\Sigma, z_{0}\right)$ (see for example Theorem 5.6 in [5]), so we will in general see $\gamma$ as an element of $\pi_{1}\left(\Sigma, z_{0}\right)$. This identification, however, is not canonical, as it depends on the choice of $\widetilde{z}_{0}$. Under this identification, the monodromy of $\Phi$ with respect to $\gamma \in \pi_{1}\left(\Sigma, z_{0}\right)$ is given by

$$
\mathcal{M}_{\gamma}(\Phi)(\lambda)=\Phi(\widetilde{\gamma}(1), \lambda) \Phi(\widetilde{\gamma}(0), \lambda)^{-1}
$$

where $\tilde{\gamma}:[0,1] \rightarrow \widetilde{\Sigma}$ is the lift of $\gamma$ such that $\widetilde{\gamma}(0)=\widetilde{z}_{0}$.

\subsection{Basic examples.}

1. A round sphere is obtained with the data

$$
\Sigma=\mathbb{C}, \quad \xi(z, \lambda)=\left(\begin{array}{cc}
0 & \lambda^{-1} \\
0 & 0
\end{array}\right) d z, \quad z_{0}=0, \quad \phi_{0}=I_{2} .
$$

The solution of the Cauchy Problem is

$$
\Phi(z, \lambda)=\left(\begin{array}{cc}
1 & \lambda^{-1} z \\
0 & 1
\end{array}\right) .
$$

Its Iwasawa decomposition is

$$
F(z, \lambda)=\frac{1}{\sqrt{1+|z|^{2}}}\left(\begin{array}{cc}
1 & \lambda^{-1} z \\
-\lambda \bar{z} & 1
\end{array}\right), \quad B(z, \lambda)=\frac{1}{\sqrt{1+|z|^{2}}}\left(\begin{array}{cc}
1 & 0 \\
\lambda \bar{z} & 1+|z|^{2}
\end{array}\right) .
$$

The Sym-Bobenko formula gives

$$
f(z)=\frac{1}{1+|z|^{2}}\left(2 \operatorname{Re}(z), 2 \operatorname{Im}(z),-2|z|^{2}\right)=(0,0,-1)+\pi^{-1}(z)
$$

where

$$
\pi^{-1}(z)=\left(\frac{2 \operatorname{Re}(z)}{1+|z|^{2}}, \frac{2 \operatorname{Im}(z)}{1+|z|^{2}}, \frac{1-|z|^{2}}{1+|z|^{2}}\right)
$$

is the inverse stereographic projection from the south pole. The immersion $f$ extends smoothly at $\infty$ and gives a conformal parametrisation of the unit sphere centered at $(0,0,-1)$.

2. Delaunay surfaces are obtained with the data

$$
\Sigma=\mathbb{C} \backslash\{0\}, \quad \xi(z, \lambda)=\left(\begin{array}{cc}
0 & r \lambda^{-1}+s \\
r \lambda+s & 0
\end{array}\right) \frac{d z}{z}, \quad z_{0}=1, \quad \phi_{0}=I_{2}
$$

where $r, s$ are non-zero real numbers such that $r+s=\frac{1}{2}$. 


\subsection{Gauging.}

Definition 1. A gauge on $\Sigma$ is a map $G: \Sigma \rightarrow \Lambda_{+} S L(2, \mathbb{C})$ such that $G(z, \lambda)$ depends holomorphically on $z \in \Sigma$ and $\lambda \in \mathbb{D}_{\rho}$ and $G(z, 0)$ is upper triangular (with no restriction on its diagonal elements).

Let $\Phi$ be a solution of $d_{z} \Phi=\Phi \xi$ and $G$ be a gauge. Let $\widehat{\Phi}=\Phi \times G$. Then $\widehat{\Phi}$ and $\Phi$ define the same immersion $f$. This is called "gauging". The gauged potential is

$$
\widehat{\xi}=\widehat{\Phi}^{-1} d_{z} \widehat{\Phi}=G^{-1} \xi G+G^{-1} d_{z} G
$$

and will be denoted $\xi \cdot G$, the dot denoting the action of the gauge group on the potential.

\section{Change of variable Depending on $\lambda$}

The following theorem allows us to use $\lambda$-dependent changes of variable in the DPW method. We will use this theorem in Section 9 with $\psi_{\lambda}$ a translation depending on $\lambda$.

Let $U$ and $V$ be Riemann surfaces, $\rho>1$ and $\psi: U \times \mathbb{D}_{\rho} \rightarrow V$ be a holomorphic map. For $(z, \lambda) \in$ $U \times \mathbb{D}_{\rho}$, we write $\psi_{\lambda}(z)=\psi(z, \lambda)$. Let $\xi(z, \lambda)$ be a DPW potential on $V$.

Theorem 3. Assume that $V$ is simply connected. Let $\Phi(z, \lambda)$ be a solution of $d_{z} \Phi=\Phi \xi$, holomorphic on $V \times \mathbb{D}_{\rho}^{*}$, and $f: V \rightarrow \mathbb{R}^{3}$ be the immersion defined by $f=\operatorname{Sym}(\operatorname{Uni}(\Phi))$. Define:

$$
\widehat{\Phi}(z, \lambda)=\Phi\left(\psi_{\lambda}(z), \lambda\right) \quad \text { for }(z, \lambda) \in U \times \mathbb{D}_{\rho}^{*} .
$$

Let $\hat{f}: U \rightarrow \mathbb{R}^{3}$ be the (branched) immersion defined by $\hat{f}=\operatorname{Sym}(\operatorname{Uni}(\widehat{\Phi}))$. Then $\hat{f}=f \circ \psi_{0}$ in $U$.

Remark 1. 1. We relax the hypothesis that $V$ is simply connected in Corollary 1 .

2. $\widehat{\Phi}$ solves $d \widehat{\Phi}=\widehat{\Phi} \hat{\xi}$ in $U$ where $\hat{\xi}(\cdot, \lambda)=\psi_{\lambda}^{*} \xi(\cdot, \lambda)$.

3. Let $(F, B)$ be the Iwasawa decomposition of $\Phi$. Then of course

$$
\widehat{\Phi}(z, \lambda)=F\left(\psi_{\lambda}(z), \lambda\right) \times B\left(\psi_{\lambda}(z), \lambda\right)
$$

but this is in general not the Iwasawa decomposition of $\widehat{\Phi}(z, \lambda)$. It is true that $F\left(\psi_{\lambda}(z), \lambda\right) \in \Lambda S U(2)$. But in general, $B(z, \lambda)$ does not depend holomorphically on $z$, so there is no reason that $B\left(\psi_{\lambda}(z), \lambda\right)$ should extend holomorphically to $\lambda \in \mathbb{D}$. For example, in the case of the spherical potential (see Section 2.5), if $B\left(\psi_{\lambda}(z), \lambda\right)$ is holomorphic with respect to $\lambda$, Equation (3) gives that $\left|\psi_{\lambda}(z)\right|$ is holomorphic, hence $\psi_{\lambda}(z)$ is constant with respect to $\lambda$.

Proof of Theorem 3, define for $z \in U$ and $\lambda \in \mathbb{D}_{\rho}^{*}$ :

$$
\widetilde{\Phi}(z, \lambda)=\Phi\left(\psi_{0}(z), \lambda\right) \text {. }
$$

Then

$$
\widetilde{\Phi}(z, \lambda)=F\left(\psi_{0}(z), \lambda\right) \times B\left(\psi_{0}(z), \lambda\right)
$$

is the Iwasawa decomposition of $\widetilde{\Phi}(z, \lambda)$. (The difference with Point 3 of Remark 1 is that $\psi_{0}(z)$ does not depend on $\lambda$.) Let $\tilde{f}=\operatorname{Sym}(\operatorname{Uni}(\widetilde{\Phi}))$. By the Sym-Bobenko formula, we obtain for $z \in U$

$$
\tilde{f}(z)=f\left(\psi_{0}(z)\right) \text {. }
$$

Define for $z \in U$ and $\lambda \in \mathbb{D}_{\rho}^{*}$ :

$$
G(z, \lambda)=\widetilde{\Phi}(z, \lambda)^{-1} \times \widehat{\Phi}(z, \lambda)=\Phi\left(\psi_{0}(z), \lambda\right)^{-1} \times \Phi\left(\psi_{\lambda}(z), \lambda\right) .
$$

The following two claims prove that $G$ is a gauge. Hence $\widetilde{\Phi}$ and $\widehat{\Phi}$ are gauge-equivalent, so $\widetilde{f}=\widehat{f}$ in $U$. This proves Theorem 3

Claim 1. $G(z, \lambda)$ extends holomorphically at $\lambda=0$. 
Proof: we use Grönwall inequality to estimate $G(z, \lambda)$. Fix $z \in U$ and $\lambda \in \mathbb{D}^{*}$. Consider the path $\gamma:[0,1] \rightarrow V$ defined by $\gamma(s)=\psi_{s \lambda}(z)$. Then for $s \in[0,1]$

$$
\frac{d}{d s} \Phi(\gamma(s), \lambda)=d_{z} \Phi(\gamma(s), \lambda) \gamma^{\prime}(s)=\Phi(\gamma(s), \lambda) \xi(\gamma(s), \lambda) \gamma^{\prime}(s) .
$$

Hence for $t \in[0,1]$

$$
\Phi(\gamma(t), \lambda)=\Phi(\gamma(0), \lambda)+\int_{0}^{t} \Phi(\gamma(s), \lambda) \xi(\gamma(s), \lambda) \gamma^{\prime}(s) d s .
$$

Multiplying by $\Phi(\gamma(0), \lambda)^{-1}$ on the left an taking norms, we obtain

$$
\left\|\Phi(\gamma(0), \lambda)^{-1} \Phi(\gamma(t), \lambda)\right\| \leq 1+\int_{0}^{t}\left\|\Phi(\gamma(0), \lambda)^{-1} \Phi(\gamma(s), \lambda)\right\| \cdot\left\|\xi(\gamma(s), \lambda) \gamma^{\prime}(s)\right\| d s .
$$

By Grönwall inequality, we obtain

$$
\|G(z, \lambda)\|=\left\|\Phi(\gamma(0), \lambda)^{-1} \Phi(\gamma(1), \lambda)\right\| \leq \exp \int_{0}^{1}\left\|\xi(\gamma(s), \lambda) \gamma^{\prime}(s)\right\| d s .
$$

We have

$$
\gamma^{\prime}(s)=\lambda \frac{\partial \psi}{\partial \lambda}(z, s \lambda)
$$

Hence

$$
\left\|\xi(\gamma(s), \lambda) \gamma^{\prime}(s)\right\|=\left\|\lambda \xi\left(\psi_{s \lambda}(z), \lambda\right) \frac{\partial \psi}{\partial \lambda}(z, s \lambda)\right\| \leq c
$$

for some constant $c$ independent of $\lambda \in \mathbb{D}^{*}$, because $\xi$ has a simple pole at $\lambda=0$ and using continuity. Hence $G(z, \lambda)$ is bounded for $\lambda \in \mathbb{D}^{*}$. By Riemann extension theorem, $G(z, \lambda)$ extends holomorphically at $\lambda=0$.

Claim 2. $G(z, 0)$ is upper triangular.

Proof: Define

$$
K(\lambda)=\left(\begin{array}{cc}
\frac{1}{\sqrt{\lambda}} & 0 \\
0 & \sqrt{\lambda}
\end{array}\right)
$$

and let $\breve{\Phi}=\Phi K$. Then $\breve{\Phi}$ solves $d_{z} \breve{\Phi}=\breve{\Phi} \check{\xi}$ where

$$
\check{\xi}=\xi \cdot K=K^{-1} \xi K \text {. }
$$

Of course, $K$ is not an admissible gauge and $\check{\xi}$ is not a DPW potential (which does not matter here). But if we write $\xi$ as in Equation (1), we have

$$
\check{\xi}=\left(\begin{array}{cc}
\alpha & \beta \\
\lambda^{-1} \gamma & -\alpha
\end{array}\right)
$$

so $\check{\xi}$ has (at most) a simple pole at $\lambda=0$. Define

$$
\breve{G}(z, \lambda)=\breve{\Phi}\left(\psi_{0}(z), \lambda\right)^{-1} \times \breve{\Phi}\left(\psi_{\lambda}(z), \lambda\right) .
$$

By the proof of Claim 1, since $\breve{\xi}$ has a simple pole at $\lambda=0, \breve{G}(z, \lambda)$ extends holomorphically at $\lambda=0$. Now $G$ and $\breve{G}$ are related by

$$
G(z, \lambda)=K(\lambda) \check{G}(z, \lambda) K(\lambda)^{-1} .
$$

This gives $G_{21}(z, \lambda)=\lambda \breve{G}_{21}(z, \lambda)$, so $G_{21}(z, 0)=0$.

Let us illustrate the proof of Theorem 3 in a case where one can compute explicitely the gauge $G$. Consider a DPW potential of the form

$$
\xi(z, \lambda)=A(\lambda) \omega(z)
$$


Then any solution $\Phi$ has the following form:

$$
\Phi(z, \lambda)=\Phi\left(z_{0}, \lambda\right) \exp \left[A(\lambda) \int_{z_{0}}^{z} \omega\right]
$$

The gauge $G$ is given by

$$
G(z, \lambda)=\exp \left[A(\lambda) \int_{\psi_{0}(z)}^{\psi_{\lambda}(z)} \omega\right]
$$

and it is straightforward to check that $G$ extends holomorphically at $\lambda=0$ with upper triangular value.

We now relax the hypothesis that $V$ is simply connected. Let $p: \widetilde{U} \rightarrow U$ and $q: \widetilde{V} \rightarrow V$ be the universal covers of $U$ and $V$. Since $\widetilde{U} \times \mathbb{D}_{\rho}$ is simply connected, the holomorphic map $\psi: U \times \mathbb{D}_{\rho} \rightarrow V$ lifts to a holomorphic map $\tilde{\psi}: \widetilde{U} \times \mathbb{D}_{\rho} \rightarrow \tilde{V}$.

Corollary 1. Let $\Phi(z, \lambda)$ be a solution of $d_{z} \Phi=\Phi \xi$ on $\tilde{V} \times \mathbb{D}_{\rho}^{*}$. Let $\tilde{f}: \tilde{V} \rightarrow \mathbb{R}^{3}$ be the immersion defined by $\tilde{f}=\operatorname{Sym}(\operatorname{Uni}(\Phi))$. Assume that $\Phi$ solves the Monodromy Problem, so $\tilde{f}$ descends to $f: V \rightarrow \mathbb{R}^{3}$. Define

$$
\widehat{\Phi}(z, \lambda)=\Phi\left(\tilde{\psi}_{\lambda}(z), \lambda\right) \quad \text { for }(z, \lambda) \in \tilde{U} \times \mathbb{D}_{\rho}^{*} .
$$

Let $\hat{f}: \widetilde{U} \rightarrow \mathbb{R}^{3}$ be the (branched) immersion defined by $\widehat{f}=\operatorname{Sym}(\operatorname{Uni}(\widehat{\Phi}))$. Then $\hat{f}=f \circ \psi_{0} \circ p$ in $\tilde{U}$. In other words, $\hat{f}$ descends to $f \circ \psi_{0}$.

Proof: by Theorem 3 , we have $\hat{f}=\tilde{f} \circ \widetilde{\psi}_{0}$ in $\widetilde{U}$. The conclusion follows from the following commutative diagram:

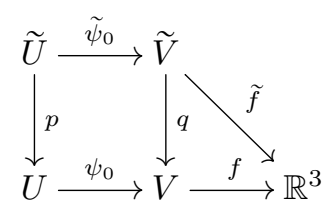

\section{Functional spaces}

In the next section, we propose a DPW potential for $n$-noids. The parameters in the definition of this potential are functions of $\lambda$. Since we plan to use the Implicit Function Theorem, we need to introduce suitable functional spaces. We decompose a function $f: \mathbb{S}^{1} \rightarrow \mathbb{C}$ in Fourier series

$$
f(\lambda)=\sum_{i \in \mathbb{Z}} f_{i} \lambda^{i}
$$

Fix some $\rho>1$ and define

$$
\|f\|=\sum_{i \in \mathbb{Z}}\left|f_{i}\right| \rho^{|i|}
$$

Let $\mathcal{W}$ be the space of functions $f$ with finite norm. This is a Banach algebra (classically called the Wiener algebra when $\rho=1$ ). Functions in $\mathcal{W}$ extend holomorphically to the annulus $\frac{1}{\rho}<|\lambda|<\rho$.

We define $\mathcal{W}^{\geq 0}, \mathcal{W}^{+}, \mathcal{W} \leq 0$ and $\mathcal{W}^{-}$as the subspaces of functions $f$ such that $f_{i}=0$ for $i<0$, $i \leq 0, i>0$ and $i \geq 0$, respectively. Functions in $\mathcal{W} \geq 0$ extend holomorphically to the disk $\mathbb{D}_{\rho}$ and satisfy $|f(\lambda)| \leq\|f\|$ for all $\lambda \in \mathbb{D}_{\rho}$. We write $\mathcal{W}^{0} \sim \mathbb{C}$ for the subspace of constant functions, so we have a direct $\operatorname{sum} \mathcal{W}=\mathcal{W}^{-} \oplus \mathcal{W}^{0} \oplus \mathcal{W}^{+}$. A function $f$ will be decomposed as $f=f^{-}+f^{0}+f^{+}$with $\left(f^{-}, f^{0}, f^{+}\right) \in \mathcal{W}^{-} \times \mathcal{W}^{0} \times \mathcal{W}^{+}$. 
We define the star operator by

$$
f^{*}(\lambda)=\overline{f\left(\frac{1}{\bar{\lambda}}\right)}=\sum_{i \in \mathbb{Z}} \overline{f_{-i}} \lambda^{i}
$$

The involution $f \mapsto f^{*}$ exchanges $\mathcal{W} \geq 0$ and $\mathcal{W} \leq 0$. We have $\lambda^{*}=\lambda^{-1}$ and $c^{*}=\bar{c}$ if $c$ is a constant. A function $f$ is real on the unit circle if and only if $f=f^{*}$.

\section{The DPW POTENTIAL}

We now start the proof of Theorem 1. Without loss of generality, we assume (by a rotation) that all vectors $u_{i}$ are non vertical. Let $\pi: \mathbb{S}^{2} \rightarrow \mathbb{C} \cup\{\infty\}$ be the stereographic projection from the south pole. For $i \in[1, n]$, we define $\pi_{i}=\pi\left(u_{i}\right) \in \mathbb{C}^{*}$ and we introduce three $\lambda$-dependent parameters $a_{i}, b_{i}$ and $p_{i}$ in the space $\mathcal{W}^{\geq 0}$. The collection of these parameters is denoted $\mathbf{x}=\left(a_{i}, b_{i}, p_{i}\right)_{1 \leq i \leq n} \in\left(\mathcal{W}^{\geq 0}\right)^{3 n}$. The parameter $\mathbf{x}$ is in a neighborhood of a (constant) central value which we denote $\mathbf{x}_{0}$ : the central value of $p_{i}$ is $\pi_{i}$, the central value of $a_{i}$ is $\tau_{i}$, and we will compute the central value of $b_{i}$ in Section 7. We define a meromorphic 1-form $\omega_{\mathbf{x}}$ on the Riemann sphere $\mathbb{C} \cup\{\infty\}$, depending on $\lambda$ and the parameter $\mathbf{x}$, by

$$
\omega_{\mathbf{x}}(z, \lambda)=\sum_{i=1}^{n}\left(\frac{a_{i}(\lambda)}{\left(z-p_{i}(\lambda)\right)^{2}}+\frac{b_{i}(\lambda)}{z-p_{i}(\lambda)}\right) d z .
$$

For $t$ in a neighborhood of 0 in $\mathbb{R}$, we define the meromorphic DPW potential $\xi_{t, \mathbf{x}}$ by

$$
\xi_{t, \mathbf{x}}(z, \lambda)=\left(\begin{array}{cc}
0 & \lambda^{-1} d z \\
t(\lambda-1)^{2} \omega_{\mathbf{x}}(z, \lambda) & 0
\end{array}\right)
$$

and take the initial condition $z_{0}=0, \phi_{0}=I_{2}$. Since the parameters $a_{i}, b_{i}$ and $p_{i}$ are holomorphic functions of $\lambda$ in the disk $\mathbb{D}_{\rho}, \xi_{t, \mathbf{x}}$ is an admissible DPW potential. Here are some of its properties:

1. If $t=0$, we get the standard DPW data for the sphere (see Section 2.5). Therefore, for small $t \neq 0$, we are constructing, away from the poles, a perturbation of the unit sphere centered at $(0,0,-1)$. We could of course have chosen the initial condition $\phi_{0}$ so that the sphere is centered at the origin: it suffices to take

$$
\phi_{0}(\lambda)=\left(\begin{array}{cc}
e^{\frac{\lambda^{2}-1}{4 \lambda}} & 0 \\
0 & e^{\frac{1-\lambda^{2}}{4 \lambda}}
\end{array}\right) \in \Lambda S U(2)
$$

but it is simpler to take $\phi_{0}=I_{2}$ and translate afterwards the immersion by $(0,0,1)$.

2. Thanks to the factor $(\lambda-1)^{2}$ in front of $\omega_{\mathbf{x}}$ in the definition of $\xi_{t, \mathbf{x}}$, Equations (ii) and (iii) of the Monodromy Problem (2) are automatically solved.

3. We will see in Section 9 that in a neighborhood of $p_{i}$, the potential $\xi_{t, \mathbf{x}}$ is gauge-equivalent to a potential with a simple pole, yielding a fuchsian system of differential equations. Moreover, provided the Monodromy Problem is solved, the residue of the gauged potential is a standard Delaunay residue. Therefore, the immersion will have Delaunay ends by the work of Kilian, Rossman and Schmitt in [17.

Remark 2. A potential of the form (7) has been introduced for 3-noids in [22]. The main difference is that in that paper, $\omega$ is fixed: the parameter used to solve the Monodromy Problem is the initial condition $\phi_{0} \in \Lambda S L(2, \mathbb{C})$, but this works only for $n=3$.

\section{The EQUATIONS}

6.1. Regularity at $\infty$. The potential $\xi_{t, \mathbf{x}}$ has a double pole at $\infty$. We want our immersion to extend smoothly at $\infty$, so we require the potential to be gauge-equivalent to a regular potential at $\infty$. Consider 
the gauge

$$
G_{\infty}(z, \lambda)=\left(\begin{array}{cc}
z & 0 \\
-\lambda & z^{-1}
\end{array}\right)
$$

The gauged potential is

$$
\xi_{t, \mathbf{x}} \cdot G_{\infty}=\left(\begin{array}{cc}
0 & \lambda^{-1} \frac{d z}{z^{2}} \\
t(\lambda-1)^{2} z^{2} \omega_{\mathbf{x}} & 0
\end{array}\right) .
$$

It is regular at $\infty$ if and only if $\omega_{\mathbf{x}}$ has a double zero at $\infty$. Using the coordinate $w=1 / z$ in a neighborhood of $\infty$, we obtain

$$
\omega_{\mathbf{x}}=-\sum_{i=1}^{n}\left(b_{i} w^{-1}+\left(a_{i}+b_{i} p_{i}\right) w^{0}+\left(2 a_{i} p_{i}+b_{i} p_{i}^{2}\right) w+O\left(w^{2}\right)\right) d w
$$

We define the following functions:

$$
\begin{gathered}
\mathcal{H}_{1}(\mathbf{x})=\sum_{i=1}^{n} b_{i} \\
\mathcal{H}_{2}(\mathbf{x})=\sum_{i=1}^{n} a_{i}+b_{i} p_{i} \\
\mathcal{H}_{3}(\mathbf{x})=\sum_{i=1}^{n} 2 a_{i} p_{i}+b_{i} p_{i}^{2} .
\end{gathered}
$$

We need to solve the equations $\mathcal{H}_{1}(\mathbf{x})=\mathcal{H}_{2}(\mathbf{x})=\mathcal{H}_{3}(\mathbf{x})=0$ so that $\omega_{\mathbf{x}}$ has a double zero at $\infty$.

6.2. The Monodromy Problem. Our potential has poles at $p_{1}, \cdots, p_{n}$ which are functions of $\lambda$. Because the DPW method requires a fixed Riemann surface, we introduce the following domain:

$$
\Omega=\left\{z \in \mathbb{C}: \forall i \in[1, n],\left|z-\pi_{i}\right|>\varepsilon\right\}
$$

where $\varepsilon>0$ is a fixed, small enough number such that the disks $D\left(\pi_{i}, 8 \varepsilon\right)$ for $1 \leq i \leq n$ are disjoint and do not contain 0 . We use the following standard notations for domains in the $z$-plane:

$$
D(p, r)=\{z \in \mathbb{C}:|z-p|<r\} \quad \text { and } \quad D^{*}(p, r)=D(p, r) \backslash\{p\} .
$$

For $\mathbf{x}$ close enough to $\mathbf{x}_{0}, \xi_{t, \mathbf{x}}$ is holomorphic in $\Omega \times \mathbb{D}_{\rho}^{*}$. Our goal is first to construct a family of immersion $f_{t}$ on $\Omega$. Then we extend $f_{t}$ to an $n$-punctured Riemann sphere in Section 9, using Corollary 1

Let $\widetilde{\Omega}$ be the universal cover of $\Omega$ and $\Phi_{t, \mathbf{x}}(z, \lambda)$ be the solution of the Cauchy Problem $d_{z} \Phi_{t, \mathbf{x}}=\Phi_{t, \mathbf{x}} \xi_{t, \mathbf{x}}$ on $\widetilde{\Omega}$ with initial condition $\Phi_{t, \mathbf{x}}(\widetilde{0}, \lambda)=I_{2}$. For $i \in[1, n]$, we denote $\gamma_{1}, \cdots, \gamma_{n}$ a set of generators of the fundamental group $\pi_{1}(\Omega, 0)$, with $\gamma_{i}$ encircling the point $\pi_{i}$ (in other words, freely homotopic in $\Omega$ to the circle $\left.C\left(\pi_{i}, 2 \varepsilon\right)\right)$. Let

$$
M_{i}(t, \mathbf{x})=\mathcal{M}_{\gamma_{i}}\left(\Phi_{t, \mathbf{x}}\right)
$$

be the monodromy of $\Phi_{t, \mathbf{x}}$ with respect to $\gamma_{i}$. Provided the Regularity Problem at $\infty$ is solved, $\Phi_{t, \mathbf{x}}$ has no monodromy around $\infty$, so we need to solve the following Monodromy Problem

$$
M_{i}(t, \mathbf{x}) \in \Lambda S U(2) \quad \text { for } 1 \leq i \leq n-1 .
$$

At $t=0$, we have $M_{i}(0, \mathbf{x})=I_{2}$. Recall that the matrix exponential is a local diffeomorphism from a neighborhood of 0 in the Lie algebra $\mathfrak{s l}(2, \mathbb{C})$ (respectively $\mathfrak{s u}(2)$ ) to a neighborhood of $I_{2}$ in $S L(2, \mathbb{C}$ ) (respectively $S U(2)$ ). The inverse diffeomorphism is denoted log. For $t \neq 0$ small enough and $\lambda \in \mathbb{D}_{\rho} \backslash\{1\}$, we define:

$$
\widetilde{M}_{i}(t, \mathbf{x})(\lambda):=\frac{\lambda}{t(\lambda-1)^{2}} \log M_{i}(t, \mathbf{x})(\lambda)
$$


Observe that $\lambda \in \mathbb{S}^{1} \Rightarrow \frac{(\lambda-1)^{2}}{\lambda} \in \mathbb{R}$. So for $t \neq 0$ and $\lambda \neq 1$, Problem (9) is equivalent to the following Rescaled Monodromy Problem:

$$
\widetilde{M}_{i}(t, \mathbf{x}) \in \Lambda \mathfrak{s u}(2) \quad \text { for } 1 \leq i \leq n-1 .
$$

The elements of the matrix $\widetilde{M}_{i}$ are denoted $\widetilde{M}_{i ; k \ell}$ for $1 \leq k, \ell \leq 2$.

Proposition 1. $\widetilde{M}_{i}(t, \mathbf{x})(\lambda)$ extends smoothly at $t=0$ and $\lambda=1$, and for $1 \leq k, \ell \leq 2, \widetilde{M}_{i ; k \ell}$ is a smooth map from a neighborhood of $\left(0, \mathbf{x}_{0}\right)$ in $\mathbb{R} \times\left(\mathcal{W}^{\geq 0}\right)^{3}$ to $\mathcal{W}$. Moreover, at $t=0$, we have

$$
\widetilde{M}_{i}(0, \mathbf{x})=2 \pi \mathrm{i}\left(\begin{array}{cc}
a_{i}+b_{i} p_{i} & -\lambda^{-1}\left(2 a_{i} p_{i}+b_{i} p_{i}^{2}\right) \\
\lambda b_{i} & -a_{i}-b_{i} p_{i}
\end{array}\right) .
$$

Proof: we first consider the case where the parameter $\mathbf{x}=\left(a_{i}, b_{i}, p_{i}\right)_{1 \leq i \leq n}$ is constant with respect to $\lambda$, so $\mathbf{x} \in \mathbb{C}^{3 n}$. Fix $R>\rho$ and let $\mathbb{A}_{R}$ be the annulus $\frac{1}{R}<|z|<R$ in $\mathbb{C}$. For $(\mu, \mathbf{x})$ in a neighborhood of $\left(0, \mathbf{x}_{0}\right)$ in $\mathbb{C} \times \mathbb{C}^{3 n}$ and $\lambda \in \mathbb{A}_{R}$, we define

$$
\xi_{\mu, \mathbf{x}, \lambda}(z)=\left(\begin{array}{cc}
0 & \lambda^{-1} d z \\
\mu \omega_{\mathbf{x}}(z) & 0
\end{array}\right)
$$

where $\omega_{\mathbf{x}}$ is defined as in Equation (6) except that $a_{i}, b_{i}, p_{i}$ are constant complex numbers. Let $\Phi_{\mu, \mathbf{x}, \lambda}(z)$ be the solution of $d \Phi_{\mu, \mathbf{x}, \lambda}=\Phi_{\mu, \mathbf{x}, \lambda} \xi_{\mu, \mathbf{x}, \lambda}$ in $\widetilde{\Omega}$ with initial condition $\Phi_{\mu, \mathbf{x}, \lambda}(\widetilde{0})=I_{2}$. Let $N_{i}(\mu, \mathbf{x}, \lambda)=$ $\mathcal{M}_{\gamma_{i}}\left(\Phi_{\mu, \xi, \lambda}\right)$. By standard O.D.E. theory, each element $N_{i ; k \ell}$ of $N_{i}$ is a holomorphic function of $\mu, \mathbf{x}$ and $\lambda$. At $\mu=0$, we have

$$
\Phi_{0, \mathbf{x}, \lambda}(z)=\left(\begin{array}{cc}
1 & \lambda^{-1} z \\
0 & 1
\end{array}\right)
$$

so $N_{i}(0, \mathbf{x}, \lambda)=I_{2}$. Hence

$$
\tilde{N}_{i}(\mu, \mathbf{x}, \lambda):=\frac{\lambda}{\mu} \log N_{i}(\mu, \mathbf{x}, \lambda)
$$

extends holomorphically at $\mu=0$ with value $\widetilde{N}_{i}(0, \mathbf{x}, \lambda)=\lambda \frac{\partial N_{i}}{\partial \mu}(0, \mathbf{x}, \lambda)$, and is holomorphic with respect to $(\mu, \mathbf{x})$ in a neighborhood of $\left(0, \mathbf{x}_{0}\right)$ and $\lambda \in \mathbb{A}_{R}$ (as a function of several complex variables). By Proposition 8 in Appendix $\mathrm{A}$

$$
\frac{\partial N_{i}}{\partial \mu}(0, \mathbf{x}, \lambda)=\left.\int_{\gamma_{i}} \Phi_{0, \mathbf{x}, \lambda} \frac{\partial \xi_{\mu, \mathbf{x}, \lambda}}{\partial \mu}\right|_{\mu=0} \Phi_{0, \mathbf{x}, \lambda}^{-1} .
$$

By the Residue Theorem, we obtain

$$
\begin{aligned}
\tilde{N}_{i}(0, \mathbf{x}, \lambda) & =2 \pi \mathrm{i} \lambda \operatorname{Res}_{p_{i}}\left(\begin{array}{cc}
1 & \lambda^{-1} z \\
0 & 1
\end{array}\right)\left(\begin{array}{cc}
0 & 0 \\
\omega_{\mathbf{x}} & 0
\end{array}\right)\left(\begin{array}{cc}
1 & -\lambda^{-1} z \\
0 & 1
\end{array}\right) \\
& =2 \pi \mathrm{i} \operatorname{Res}_{p_{i}}\left(\begin{array}{cc}
z & -\lambda^{-1} z^{2} \\
\lambda & -z
\end{array}\right)\left(\frac{a_{i}}{\left(z-p_{i}\right)^{2}}+\frac{b_{i}}{z-p_{i}}\right) \\
& =2 \pi \mathrm{i}\left(\begin{array}{cc}
a_{i}+b_{i} p_{i} & -\lambda^{-1}\left(2 a_{i} p_{i}+b_{i} p_{i}^{2}\right) \\
\lambda b_{i} & -a_{i}-b_{i} p_{i}
\end{array}\right) .
\end{aligned}
$$

In the last equation, we have used the following elementary residue computation:

$$
\operatorname{Res}_{p} \frac{z^{k}}{(z-p)^{2}}=k p^{k-1}
$$

For $\mathbf{x} \in\left(\mathcal{W}^{\geq 0}\right)^{3 n}$, we have

$$
\begin{gathered}
\xi_{t, \mathbf{x}}(z, \lambda)=\xi_{t(\lambda-1)^{2}, \mathbf{x}(\lambda), \lambda}(z) \\
\widetilde{M}_{i}(t, \mathbf{x})(\lambda)=\widetilde{N}_{i}\left(t(\lambda-1)^{2}, \mathbf{x}(\lambda), \lambda\right) .
\end{gathered}
$$


Since the linear map $(t, \mathbf{x}) \mapsto\left(t(\lambda-1)^{2}, \mathbf{x}\right)$ from $\mathbb{R} \times\left(\mathcal{W}^{\geq 0}\right)^{3 n}$ to $\mathcal{W}^{3 n+1}$ is bounded, Proposition 9 in Appendix $\mathrm{B}$ gives that for $1 \leq k, \ell \leq 2$, the map $(t, \mathbf{x}) \mapsto \widetilde{M}_{i ; k \ell}(t, \mathbf{x})$ is smooth from a neighborhood of $\left(0, \mathbf{x}_{0}\right)$ in $\mathbb{R} \times\left(\mathcal{W}^{\geq 0}\right)^{3 n}$ to $\mathcal{W}$.

Remark 3. Proposition 1 implies in particular that $M_{i}(t, \mathbf{x})(1)=0$ and $\frac{\partial M_{i}(t, \mathbf{x})}{\partial \lambda}(1)=0$ as claimed in Point 2 of Section 5 .

We define the following functions (the $*$ operator is defined in Section 4):

$$
\begin{gathered}
\mathcal{F}_{i}(t, \mathbf{x})=\widetilde{M}_{i, 11}(t, \mathbf{x})+\widetilde{M}_{i, 11}(t, \mathbf{x})^{*} \\
\mathcal{G}_{i}(t, \mathbf{x})=\lambda\left(\widetilde{M}_{i, 12}(t, \mathbf{x})+\widetilde{M}_{i, 21}(t, \mathbf{x})^{*}\right) .
\end{gathered}
$$

The Regularity and Rescaled Monodromy Problems are equivalent to the following problem:

$$
\begin{cases}\mathcal{F}_{i}(t, \mathbf{x})=0 & \text { for } 1 \leq i \leq n-1 \\ \mathcal{G}_{i}(t, \mathbf{x})=0 & \text { for } 1 \leq i \leq n-1 \\ \mathcal{H}_{i}(\mathbf{x})=0 & \text { for } 1 \leq i \leq 3\end{cases}
$$

7. Solving the EQUATions at $t=0$

Proposition 2. When $t=0$, Problem (11) is equivalent to the following conditions, for $1 \leq i \leq n$ :

(i) $a_{i} \in \mathbb{R}$ is constant (with respect to $\lambda$ ).

(ii) $p_{i}$ is constant.

(iii) $b_{i}=\frac{-2 a_{i} \overline{p_{i}}}{1+\left|p_{i}\right|^{2}}$.

(iv) $\sum_{i=1}^{n} a_{i} \pi^{-1}\left(p_{i}\right)=0$ where $\pi^{-1}$ is the inverse stereographic projection from the south pole given by Equation (4).

Proof: Using Proposition 1, we obtain:

$$
\begin{gathered}
\mathcal{F}_{i}(0, \mathbf{x})=2 \pi \mathrm{i}\left(\left(a_{i}+b_{i} p_{i}\right)-\left(a_{i}+b_{i} p_{i}\right)^{*}\right) \\
\mathcal{G}_{i}(0, \mathbf{x})=-2 \pi \mathrm{i}\left(2 a_{i} p_{i}+b_{i} p_{i}^{2}+b_{i}^{*}\right) \\
\sum_{i=1}^{n} \mathcal{F}_{i}(0, \mathbf{x})=2 \pi \mathrm{i}\left(\mathcal{H}_{2}(\mathbf{x})-\mathcal{H}_{2}(\mathbf{x})^{*}\right) \\
\sum_{i=1}^{n} \mathcal{G}_{i}(0, \mathbf{x})=-2 \pi \mathrm{i}\left(\mathcal{H}_{3}(\mathbf{x})+\mathcal{H}_{1}(\mathbf{x})^{*}\right) .
\end{gathered}
$$

Assume that $t=0$ and let $\mathbf{x}=\left(a_{i}, b_{i}, p_{i}\right)_{1 \leq i \leq n}$ be a solution of Problem (11). From Equations (14) and (15) we infer that $\mathcal{F}_{n}(0, \mathbf{x})=\mathcal{G}_{n}(0, \mathbf{x})=0$. Let $i \in[1, n]$. From Equation (13), we see that $b_{i} \in \mathcal{W}^{\leq 0} \cap \mathcal{W}^{\geq 0}=\mathcal{W}^{0}$ hence $b_{i}$ is constant. From Equation 12 , we obtain by the same argument

$$
a_{i}+b_{i} p_{i}=c_{i}
$$

where $c_{i}$ is a real constant. Eliminating $a_{i}$ from Equations $(13)$ and $(16)$, we obtain

$$
-b_{i} p_{i}^{2}+2 c_{i} p_{i}+\overline{b_{i}}=0 \text {. }
$$

If $b_{i} \neq 0$ then $p_{i}$ can take only two values. Being a holomorphic function of $\lambda, p_{i}$ must be constant. By Equation (16), $a_{i}$ is constant. (If $b_{i}=0$, then $a_{i}=c_{i}$ and since $a_{i} \neq 0$, Equation (13) implies that $p_{i}=0$ is constant.) Multipliying (13) by $\overline{p_{i}}$ we obtain

$$
\left(2 a_{i}+b_{i} p_{i}\right)\left|p_{i}\right|^{2}+\overline{b_{i} p_{i}}=0 .
$$


Taking the imaginary part and using $\operatorname{Im}\left(b_{i} p_{i}\right)=-\operatorname{Im}\left(a_{i}\right)$, we obtain

$$
\operatorname{Im}\left(a_{i}\right)\left(\left|p_{i}\right|^{2}+1\right)=0 .
$$

Hence $a_{i} \in \mathbb{R}$ and so $b_{i} p_{i} \in \mathbb{R}$. Equation (17) gives

With this value for $b_{i}$ we obtain

$$
b_{i}=\frac{-2 a_{i} \overline{p_{i}}}{1+\left|p_{i}\right|^{2}} .
$$

$$
\begin{gathered}
\mathcal{H}_{1}=\sum_{i=1}^{n} \frac{-2 a_{i} \overline{p_{i}}}{1+\left|p_{i}\right|^{2}} \\
\mathcal{H}_{2}=\sum_{i=1}^{n} \frac{a_{i}\left(1-\left|p_{i}\right|^{2}\right)}{1+\left|p_{i}\right|^{2}} \\
\mathcal{H}_{3}=\sum_{i=1}^{n} \frac{2 a_{i} p_{i}}{1+\left|p_{i}\right|^{2}} .
\end{gathered}
$$

so the equations $\mathcal{H}_{2}=0$ and $\mathcal{H}_{3}=0$ give Point (iv).

Conversely, assume that $a_{i}, b_{i}$ and $p_{i}$ satisfy Points (i) to (iv) of Proposition 2. Proposition 1 gives

$$
\widetilde{M}_{i}(0, \mathbf{x})=2 \pi \mathrm{i} \frac{a_{i}}{\left(1+\left|p_{i}\right|^{2}\right)}\left(\begin{array}{cc}
1-\left|p_{i}\right|^{2} & -2 \lambda^{-1} p_{i} \\
-2 \lambda \overline{p_{i}} & \left|p_{i}\right|^{2}-1
\end{array}\right) \in \Lambda \mathfrak{s u}(2) .
$$

Equations [18), 19] and 20] imply that $\mathcal{H}_{1}=\mathcal{H}_{2}=\mathcal{H}_{3}=0$.

\section{Solving the equations using the Implicit Function Theorem}

We shall apply the Implicit Function Theorem at the point $(t, \mathbf{x})=\left(0, \mathbf{x}_{0}\right)$ where $\mathbf{x}_{0}$ denotes the following value of the parameters:

$$
a_{i}=\tau_{i}, \quad b_{i}=\frac{-2 a_{i} \overline{\pi_{i}}}{1+\left|\pi_{i}\right|^{2}} \quad \text { and } \quad p_{i}=\pi_{i} \quad \text { for } i \in[1, n] .
$$

According to Proposition 2 and by the balancing condition of Theorem 1. Problem (11) is solved at $\left(0, \mathbf{x}_{0}\right)$.

Proposition 3. For $t$ in a neighborhood of 0 , there exists a unique smooth map $t \mapsto \mathbf{x}(t)=\left(a_{i, t}, b_{i, t}, p_{i, t}\right)_{1 \leq i \leq n}$ with value in $\left(\mathcal{W}^{\geq 0}\right)^{3 n}$ such that $\mathbf{x}(0)=\mathbf{x}_{0}$, Problem (11) is solved at $(t, \mathbf{x}(t))$ and the following normalisation holds:

$$
\forall i \in[1, n-1], \quad \operatorname{Re}\left(a_{i, t}^{0}\right)=\tau_{i} \quad \text { and } \quad p_{i, t}^{0}=\pi_{i} .
$$

Proof: We compute the partial differential of Equations 12 and 13 with respect to $\mathbf{x}$ :

$$
\begin{gathered}
d_{\mathbf{x}} \mathcal{F}_{i}\left(0, \mathbf{x}_{0}\right)=2 \pi \mathrm{i}\left(d a_{i}+p_{i} d b_{i}+b_{i} d p_{i}\right)-2 \pi \mathrm{i}\left(d a_{i}+p_{i} d b_{i}+b_{i} d p_{i}\right)^{*} \\
d_{\mathbf{x}} \mathcal{G}_{i}\left(0, \mathbf{x}_{0}\right)=-2 \pi \mathrm{i}\left(2 p_{i} d a_{i}+p_{i}^{2} d b_{i}+2\left(a_{i}+b_{i} p_{i}\right) d p_{i}+d b_{i}^{*}\right) .
\end{gathered}
$$

Here, $a_{i}, b_{i}, p_{i}$ are given by Equation (21) so are constant with respect to $\lambda$. Projecting on $\mathcal{W}^{+}, \mathcal{W}^{0}$ and $\mathcal{W}^{-}$we obtain:

$$
\begin{gathered}
d_{\mathbf{x}} \mathcal{F}_{i}\left(0, \mathbf{x}_{0}\right)^{+}=2 \pi \mathrm{i}\left(d a_{i}^{+}+p_{i} d b_{i}^{+}+b_{i} d p_{i}^{+}\right) \\
d_{\mathbf{x}} \mathcal{F}_{i}\left(0, \mathbf{x}_{0}\right)^{0}=-4 \pi \operatorname{Im}\left(d a_{i}^{0}+p_{i} d b_{i}^{0}+b_{i} d p_{i}^{0}\right) \\
d_{\mathbf{x}} \mathcal{G}_{i}\left(0, \mathbf{x}_{0}\right)^{+}=-2 \pi \mathrm{i}\left(2 p_{i} d a_{i}^{+}+p_{i}^{2} d b_{i}^{+}+2\left(a_{i}+b_{i} p_{i}\right) d p_{i}^{+}\right) \\
d_{\mathbf{x}} \mathcal{G}_{i}\left(0, \mathbf{x}_{0}\right)^{0}=-2 \pi \mathrm{i}\left(2 p_{i} d a_{i}^{0}+p_{i}^{2} d b_{i}^{0}+2\left(a_{i}+b_{i} p_{i}\right) d p_{i}^{0}+\overline{d b_{i}^{0}}\right) \\
d_{\mathbf{x}} \mathcal{G}_{i}\left(0, \mathbf{x}_{0}\right)^{-}=-2 \pi \mathrm{i}\left(d b_{i}^{+}\right)^{*} \\
\left(d_{\mathbf{x}} \mathcal{G}_{i}\left(0, \mathbf{x}_{0}\right)^{-}\right)^{*}=2 \pi \mathrm{i} d b_{i}^{+} .
\end{gathered}
$$


By definition, we have $\mathcal{F}_{i}(t, \mathbf{x})=\mathcal{F}_{i}^{*}(t, \mathbf{x})$ for all $(t, \mathbf{x})$, so $\mathcal{F}_{i}^{0}(t, \mathbf{x}) \in \mathbb{R}$ and $\mathcal{F}_{i}^{-}(t, \mathbf{x})=0 \Leftrightarrow \mathcal{F}_{i}^{+}(t, \mathbf{x})=0$. We restrict the parameter $\mathbf{x}$ to the subspace defined by Equation 22 .

Claim 3. 1. For $1 \leq i \leq n-1$, the partial differential of $\left(\mathcal{F}_{i}^{+}, \mathcal{G}_{i}^{+},\left(\mathcal{G}_{i}^{-}\right)^{*}\right)$ with respect to $\left(a_{i}^{+}, b_{i}^{+}, p_{i}^{+}\right)$is an automorphism of $\left(\mathcal{W}^{+}\right)^{3}$.

2. For $1 \leq i \leq n-1$, the partial differential of $\left(\mathcal{F}_{i}^{0}, \mathcal{G}_{i}^{0}\right)$ with respect to $\left(\operatorname{Im}\left(a_{i}^{0}\right), b_{i}^{0}\right)$ is an automorphism of $\mathbb{R} \times \mathbb{C}$.

3. The partial differential of $\left(\mathcal{H}_{1}, \mathcal{H}_{2}, \mathcal{H}_{3}\right)$ with respect to $\left(a_{n}, b_{n}, p_{n}\right)$ is an automorphism of $(\mathcal{W} \geq 0)^{3}$.

Proof:

1. We can write in matrix form

$$
\left(\begin{array}{c}
d_{\mathbf{x}} \mathcal{F}_{i}^{+} \\
d_{\mathbf{x}} \mathcal{G}_{i}^{+} \\
\left(d_{\mathbf{x}} \mathcal{G}_{i}^{-}\right)^{*}
\end{array}\right)=2 \pi \mathrm{i}\left(\begin{array}{ccc}
1 & p_{i} & b_{i} \\
-2 p_{i} & -p_{i}^{2} & -2\left(a_{i}+b_{i} p_{i}\right) \\
0 & 1 & 0
\end{array}\right)\left(\begin{array}{c}
d a_{i}^{+} \\
d b_{i}^{+} \\
d p_{i}^{+}
\end{array}\right) .
$$

This constant matrix has determinant $2 a_{i}$ so is invertible. (Observe that this operator admits a matrix with respect to a decomposition of the space as a finite product of Banach spaces. It is clear that if the matrix is invertible, the operator is an automorphism.)

2. At $t=0$ and for fixed value of $\operatorname{Re}\left(a_{i}\right)$ and $p_{i},\left(\mathcal{F}_{i}(0, \mathbf{x})^{0}, \mathcal{G}_{i}(0, \mathbf{x})^{0}\right)$ is an affine function of $\left(\operatorname{Im}\left(a_{i}^{0}\right), b_{i}^{0}\right)$. The proof of Proposition 2 shows that this function is injective, so its linear part is an automorphism of $\mathbb{R} \times \mathbb{C}$.

3. The partial differential of $\left(\mathcal{H}_{1}, \mathcal{H}_{2}, \mathcal{H}_{3}\right)$ with respect to $\mathbf{y}=\left(a_{n}, b_{n}, p_{n}\right)$ can be written in matrix form as

$$
\left(\begin{array}{c}
d_{\mathbf{y}} \mathcal{H}_{1} \\
d_{\mathbf{y}} \mathcal{H}_{2} \\
d_{\mathbf{y}} \mathcal{H}_{3}
\end{array}\right)=\left(\begin{array}{ccc}
0 & 1 & 0 \\
1 & p_{n} & b_{n} \\
2 p_{n} & p_{n}^{2} & 2\left(a_{n}+b_{n} p_{n}\right)
\end{array}\right)\left(\begin{array}{c}
d a_{n} \\
d b_{n} \\
d p_{n}
\end{array}\right) .
$$

This matrix has determinant $-2 a_{n} \neq 0$.

Returning to the proof of Proposition 3 , the partial differential of

$$
\left[\left(\mathcal{F}_{i}^{+}, \mathcal{G}_{i}^{+},\left(\mathcal{G}_{i}^{-}\right)^{*}, \mathcal{F}_{i}^{0}, \mathcal{G}_{i}^{0}\right)_{1 \leq i \leq n-1}, \mathcal{H}_{1}, \mathcal{H}_{2}, \mathcal{H}_{3}\right]
$$

with respect to

$$
\left[\left(a_{i}^{+}, b_{i}^{+}, p_{i}^{+}, \operatorname{Im}\left(a_{i}^{0}\right), b_{i}^{0}\right)_{1 \leq i \leq n-1}, a_{n}, b_{n}, p_{n}\right]
$$

has lower triangular block form, with automorphisms on the diagonal so is an automorphism. (Here I am not talking about a matrix block decomposition but about the decomposition of an operator with respect to a product of Banach spaces.) Proposition 3 follows from the Implicit Function Theorem.

\section{Delaunay ends}

From now on, we assume that the parameter $\mathbf{x}=\mathbf{x}(t)$ is given as a function of $t$ by Proposition 3 . We denote $\xi_{t}=\xi_{t, \mathbf{x}(t)}, \Phi_{t}=\Phi_{t, \mathbf{x}(t)}$ and $f_{t}=\operatorname{Sym}\left(\operatorname{Uni}\left(\Phi_{t}\right)\right)$ the immersion obtained by the DPW method on $\widetilde{\Omega}$. Since the Monodromy Problem is solved, $f_{t}$ descends to a well defined immersion in $\Omega$, still denoted the same. In this section we prove:

Proposition 4. For $t \neq 0$ small enough:

1. $f_{t}$ extends analytically to

$$
\Sigma_{t}:=\mathbb{C} \cup\{\infty\} \backslash\left\{p_{1, t}(0), \cdots, p_{n, t}(0)\right\} .
$$

2. For $i \in[1, n], a_{i, t}$ is a real constant (with respect to $\lambda$ ).

3. For $i \in[1, n], f_{t}$ has a Delaunay end of weight $8 \pi t a_{i, t}$ at $p_{i, t}(0)$.

Proof: 
1. Since the Regularity Problem is solved, the immersion $f_{t}$ extends analytically at $\infty$. With the notations of Theorem 3, we consider the change of variable

$$
z=\psi_{i, t, \lambda}(w)=p_{i, t}(\lambda)+w
$$

and the following domains:

$$
\begin{gathered}
U=\{w \in \mathbb{C}: 2 \varepsilon<|w|<4 \varepsilon\} \\
V_{i}=\left\{z \in \mathbb{C}: \varepsilon<\left|z-\pi_{i}\right|<8 \varepsilon\right\} \subset \Omega
\end{gathered}
$$

For $t$ small enough, we have $\psi_{i, t, \lambda}(U) \subset V_{i}$ for all $\lambda \in \mathbb{D}_{\rho}$. Let $\tilde{U}$ and $\tilde{V}_{i}$ be the universal covers of $U$ and $V_{i}$. (For $\tilde{V}_{i}$, we may take an arbitrary component of $\widetilde{\Omega} \cap p^{-1}\left(V_{i}\right)$ where $p: \widetilde{\Omega} \rightarrow \Omega$ is the universal cover.) Lift $\psi_{i, t, \lambda}$ to $\widetilde{\psi}_{i, t, \lambda}: \widetilde{U} \rightarrow \widetilde{V}_{i}$ and define for $w \in \widetilde{U}$ :

$$
\widehat{\Phi}_{i, t}(w, \lambda)=\Phi_{t}\left(\widetilde{\psi}_{i, t, \lambda}(w), \lambda\right) .
$$

By Corollary 1, $\hat{f}_{i, t}=\operatorname{Sym}\left(\operatorname{Uni}\left(\widehat{\Phi}_{i, t}\right)\right)$ descends to a well defined immersion on $U$ and

$$
\forall w \in U, \quad \widehat{f}_{i, t}(w)=f_{t}\left(\psi_{i, t, 0}(w)\right)=f_{t}\left(p_{i, t}(0)+w\right) .
$$

Now $\widehat{\Phi}_{i, t}$ solves $d \widehat{\Phi}_{i, t}=\widehat{\Phi}_{i, t} \widehat{\xi}_{i, t}$ with

$$
\hat{\xi}_{i, t}=\psi_{i, t, \lambda}^{*} \xi_{t}=\left(\begin{array}{cc}
0 & \lambda^{-1} d w \\
t(\lambda-1)^{2} \widehat{\omega}_{i, t} & 0
\end{array}\right) \quad \text { and } \quad \hat{\omega}_{i, t}=\psi_{i, t, \lambda}^{*} \omega_{\mathbf{x}(t)} .
$$

Since the only pole of $\widehat{\omega}_{i, t}$ in $D(0,4 \varepsilon)$ is at $w=0$, the DPW method shows that $\widehat{f}_{i, t}$ extends analytically to $D^{*}(0,4 \varepsilon)$. We may extend $f_{t}$ analytically by requesting that Equation 24 holds true in $D^{*}(0,4 \varepsilon)$. Doing this for $i \in[1, n]$, we have extended $f_{t}$ analytically to $\Sigma_{t}$.

2. Next we gauge $\widehat{\xi}_{i, t}$ so that it has a simple pole at 0 , yielding a fuchsian system of differential equations. Consider the gauge

$$
G(w, \lambda)=\left(\begin{array}{cc}
\frac{\sqrt{w}}{k_{\lambda}} & 0 \\
-\frac{k}{2 k \sqrt{w}} & \frac{k}{\sqrt{w}}
\end{array}\right) .
$$

Here we can take $k=1$, but in the next point we will take another value of $k$ so we do the computation for general values of $k \neq 0$. The gauged potential is

$$
\check{\xi}_{i, t}:=\widehat{\xi}_{i, t} \cdot G=\left(\begin{array}{cc}
0 & \lambda^{-1} k^{2} \frac{d w}{w} \\
\frac{t(\lambda-1)^{2}}{k^{2}} w \widehat{\omega}_{i, t}+\frac{\lambda}{4 k^{2}} \frac{d w}{w} & 0
\end{array}\right) .
$$

It has a simple pole at 0 with residue

$$
A_{i, t}(\lambda):=\left(\begin{array}{cc}
0 & \lambda^{-1} k^{2} \\
\frac{t(\lambda-1)^{2}}{k^{2}} a_{i, t}(\lambda)+\frac{\lambda}{4 k^{2}} & 0
\end{array}\right) .
$$

The eigenvalues of $A_{i, t}(\lambda)$ are $\pm \Lambda_{i, t}(\lambda)$ with

$$
\Lambda_{i, t}(\lambda)^{2}=t \lambda^{-1}(\lambda-1)^{2} a_{i, t}(\lambda)+\frac{1}{4} .
$$

Fix $\lambda \in \mathbb{S}^{1} \backslash\{1\}$. If $t \neq 0$ is small enough, then $\Lambda_{i, t}(\lambda) \notin \mathbb{Z} / 2$ so the corresponding fuchsian system is non-resonant. Hence $\breve{\Phi}_{i, t}:=\widehat{\Phi}_{i, t} G$ has the following standard $z^{A} P$ form in the universal cover of $D(0, \varepsilon)^{*}$ (see Proposition 11.2 in [23])

$$
\breve{\Phi}_{i, t}(w, \lambda)=V(\lambda) \exp \left(A_{i, t}(\lambda) \log w\right) P(w, \lambda)
$$

where $V \in \Lambda S L(2, \mathbb{C})$ and $P(w, \lambda)$ descends to a well defined holomorphic function of $w$ in $D(0, \varepsilon)$ with $P(0, \lambda)=I_{2}$. Hence

$$
\mathcal{M}_{C(0, \varepsilon)}\left(\breve{\Phi}_{i, t}\right)(\lambda)=V(\lambda) \exp \left(2 \pi \mathrm{i} A_{i, t}(\lambda)\right) V(\lambda)^{-1}
$$


so the eigenvalues of $\mathcal{M}_{\gamma_{i}}\left(\Phi_{t}\right)$ are $\exp \left( \pm 2 \pi \mathrm{i} \Lambda_{i, t}(\lambda)\right)$. Since the Monodromy Problem is solved, the eigenvalues are unitary complex numbers, so $\Lambda_{i, t}(\lambda) \in \mathbb{R}$ which implies $a_{i, t}(\lambda) \in \mathbb{R}$. Since $a_{i, t}$ is holomorphic in $\mathbb{D}_{\rho}$ and $a_{i, t}$ is real on $\mathbb{S}^{1} \backslash\{1\}$, it is constant.

3. It remains to choose $k$ in the definition of $G$ such that $A_{i, t}(\lambda)$ is a standard Delaunay residue. Fix a small $t>0$ and let $(r, s) \in \mathbb{R}^{2}$ be the solution of the system

$$
\left\{\begin{array}{l}
r s=t a_{i, t} \\
r+s=\frac{1}{2} \\
r>s
\end{array}\right.
$$

For small $t,(r, s)$ is close to $\left(\frac{1}{2}, 0\right)$ so $\sqrt{r+s \lambda}$ is well defined and does not vanish in $\mathbb{D}_{\rho}$. We take

$$
k=\sqrt{r+s \lambda}
$$

in the definition of $G$. Using $r+s=\frac{1}{2}$, we have

$$
(r+s \lambda)(r \lambda+s)=r s(\lambda-1)^{2}+\frac{\lambda}{4}=t a_{i, t}(\lambda-1)^{2}+\frac{\lambda}{4} .
$$

Hence by Equation (25),

$$
A_{i, t}(\lambda)=\left(\begin{array}{cc}
0 & r \lambda^{-1}+s \\
r \lambda+s & 0
\end{array}\right)
$$

which is the residue of the standard Delaunay potential (see Section 2.5). Since the Monodromy Problem is solved, the immersion $\widehat{f}_{i, t}$ has a Delaunay end at $w=0$ of weight $8 \pi r s=8 \pi t a_{i, t}$ by Theorem 3.5 in [17].

Remark 4. We have constructed a smooth family of immersions $f_{t}$ defined for $t \neq 0$ in a neighborhood of 0 . As $t$ changes sign, the behavior of each end switches from unduloid to nodoid type. As $t \rightarrow 0$, the immersion $f_{t}$ degenerates into a sphere.

\section{Geometry of the immersion}

Theorem 3.5 of [17] tells us that for each $t>0$, there exists a Delaunay immersion $f_{i, t}^{\mathcal{D}}: \mathbb{C}^{*} \rightarrow \mathbb{R}^{3}$ such that

$$
\lim _{w \rightarrow 0}\left\|\hat{f}_{i, t}(w)-f_{i, t}^{\mathcal{D}}(w)\right\|=0
$$

In that paper, $t$ is fixed. The problem is that the limit $w \rightarrow 0$ is not uniform with respect to $t$. Indeed, the fuchsian system is resonant at $t=0$ so the constants in their estimates are uncontroled as $t \rightarrow 0$. Thomas Raujouan has improved this result in [20] and was able to obtain a uniform limit under additional assumptions:

Theorem 4. 20] Let $\tau$ be a non-zero real number. Let $\xi_{t}(z, \lambda)$ be a family of DPW potentials depending on the parameter $t \geq 0$ and defined for $z$ in a punctured neighborhood of 0 . Let $\Phi_{t}(z, \lambda)$ be a solution of $d_{z} \Phi_{t}=\Phi_{t} \xi_{t}$ and $f_{t}(z)=\operatorname{Sym}\left(\operatorname{Uni}\left(\Phi_{t}\right)\right)$. Assume that:

1. $\xi_{t}=A_{t} \frac{d z}{z}+O\left(t, z^{0}\right)$ where $A_{t}$ is the standard Delaunay residue given by Equation 28 and $(r, s) \in \mathbb{R}^{2}$ is the solution of

$$
\left\{\begin{array}{l}
r s=t \tau \\
r+s=\frac{1}{2} \\
r>s
\end{array}\right.
$$

2. The monodromy of $\Phi_{t}$ around the origin is in $\Lambda S U(2)$.

3. $\Phi_{0}(1, \lambda)=\left(\begin{array}{cc}a & \lambda^{-1} b \\ \lambda c & d\end{array}\right)$ where $a, b, c, d$ are (constant) complex numbers.

Then: 
1. For all $0<\alpha<1$, there exists uniform positive numbers $\epsilon, c, T$ and a family of Delaunay immersions $f_{t}^{\mathcal{D}}: \mathbb{C}^{*} \rightarrow \mathbb{R}^{3}$ with weight $8 \pi \tau t$ such that for all $0<t<T$ and $0<|z|<\epsilon$,

$$
|| f_{t}(z)-f_{t}^{\mathcal{D}}(z) \| \leq c t|z|^{\alpha} .
$$

2. Let

$$
\begin{aligned}
H(\lambda) & =\frac{1}{\sqrt{2}}\left(\begin{array}{cc}
1 & -\lambda^{-1} \\
\lambda & 1
\end{array}\right) \\
Q & =\operatorname{Uni}\left(\Phi_{0}(1, \cdot) H\right) .
\end{aligned}
$$

The axis of $f_{t}^{\mathcal{D}}$ (oriented from the end at $\infty$ to the end at 0 ) converges as $t \rightarrow 0$ to the line through the point $\operatorname{Sym}(Q)$ spanned by the vector $-\operatorname{Nor}(Q)$ (notations as in Section 2.3).

3. If $\tau>0$, there exists $T^{\prime}<T$ such that the restriction of $f_{t}$ to $D^{*}(0, \epsilon)$ is an embedding for all $0<$ $t<T^{\prime}$. More precisely, $f_{t}\left(D^{*}(0, \epsilon)\right)$ is included in an embedded tubular neighborhood of the Delaunay surface $f_{t}^{\mathcal{D}}\left(\mathbb{C}^{*}\right)$ and the projection on the Delaunay surface is a diffeomorphism from $f_{t}\left(D^{*}(0, \epsilon)\right)$ onto its image.

Observe that Hypothesis 1 implies that $\xi_{0}=A_{0} \frac{d z}{z}$ so $\Phi_{0}$ is actually defined for $z \in \mathbb{C}^{*}$, which is why Hypothesis 3 makes sense.

10.1. Axes of the ends. We first use Theorem 4 to compute the limit axes of the ends.

Proposition 5. The axis of the Delaunay end of $f_{t}$ at $p_{i, t}(0)$ converges as $t \rightarrow 0$ to the half-line through $(0,0,-1)$ spanned by the vector $u_{i}$.

Proof: we continue with the notations of the proof of Proposition 4 . We want to apply Theorem 4 to $\breve{\Phi}_{i, t}=\widehat{\Phi}_{i, t} G$. First of all, $t \mapsto t a_{i, t}$ is a smooth diffeomorphism in a neighborhood of 0 so we may use $\left|t a_{i, t}\right|$ as the time parameter in our application of Theorem 4 with $\tau=1$ if $t a_{i, t}>0$ and $\tau=-1$ if $t a_{i, t}<0$. We have

$$
\widehat{\Phi}_{i, 0}(w, \lambda)=\Phi_{0}\left(\pi_{i}+w, \lambda\right)=\left(\begin{array}{cc}
1 & \lambda^{-1}\left(\pi_{i}+w\right) \\
0 & 1
\end{array}\right) .
$$

At $t=0$, the solution of 26 is $(r, s)=\left(\frac{1}{2}, 0\right)$ so $k=\frac{1}{\sqrt{2}}$. This gives

$$
\begin{aligned}
\breve{\Phi}_{i, 0}(1, \lambda) & =\widehat{\Phi}_{i, 0}(1, \lambda) G(1, \lambda) \\
& =\left(\begin{array}{cc}
1 & \lambda^{-1}\left(\pi_{i}+1\right) \\
0 & 1
\end{array}\right)\left(\begin{array}{cc}
\sqrt{2} & 0 \\
-\frac{\lambda}{\sqrt{2}} & \frac{1}{\sqrt{2}}
\end{array}\right) \\
& =\frac{1}{\sqrt{2}}\left(\begin{array}{cc}
1-\pi_{i} & \lambda^{-1}\left(\pi_{i}+1\right) \\
-\lambda & 1
\end{array}\right) .
\end{aligned}
$$

Fix some $\alpha \in(0,1)$. By Theorem 4 , there exists uniform positive numbers $\epsilon, c, T$ and a family of Delaunay immersions $f_{i, t}^{\mathcal{D}}$ such that for $0<t<T$ and $0<|w|<\epsilon$

$$
\left\|\widehat{f}_{i, t}(w)-f_{i, t}^{\mathcal{D}}(w)\right\| \leq c t|w|^{\alpha} .
$$

Using Equation (29), the matrix $Q$ in Point 2 of Theorem 4 is given by

$$
Q(\lambda)=\operatorname{Uni}\left(\begin{array}{cc}
1 & \lambda^{-1} \pi_{i} \\
0 & 1
\end{array}\right) .
$$

By the results in Section 2.5 for the standard sphere, the limit axis of $f_{i, t}^{\mathcal{D}}$ is the line through the point $(0,0,-1)+\pi^{-1}\left(\pi_{i}\right)$ directed by the vector $\pi^{-1}\left(\pi_{i}\right)$. Since $\pi_{i}=\pi\left(u_{i}\right)$, this proves Proposition 5 


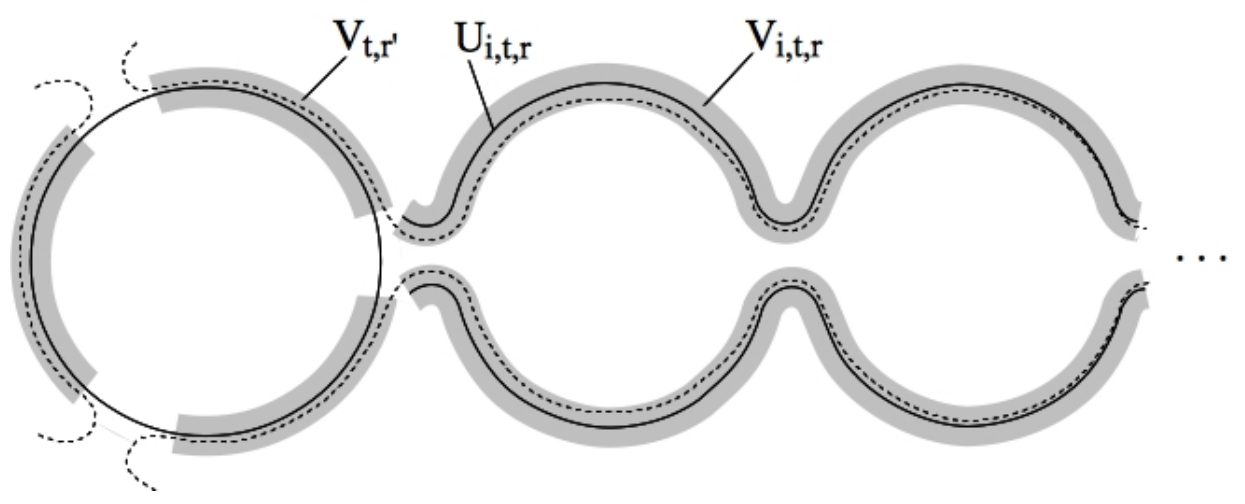

FiguRE 2. The domains $V_{i, t, r}$ and $V_{t, r^{\prime}}$. On this figure, $n=3$ and $r^{\prime}>r$ so the domains $V_{i, t, r}$ and $V_{t, r^{\prime}}$ do not overlap (for clarity). The surface $M_{t}$ is represented with dots. The Delaunay surface $\mathcal{D}_{i, t}$ and the sphere $\mathbb{S}^{2}$ are represented with plain lines.

10.2. Embeddedness. For ease of notation, we forget from now on the argument $\lambda=0$ so we write $p_{i, t}=p_{i, t}(0)$. It will be convenient to translate all immersions by the vector $(0,0,1)$, so we replace $f_{t}$ by $f_{t}+(0,0,1)$ and $f_{i, t}^{\mathcal{D}}$ by $f_{i, t}^{\mathcal{D}}+(0,0,1)$. Thanks to this translation, the standard spherical data (see Section 2.5 yields an immersion $f_{0}: \mathbb{C} \cup\{\infty\} \rightarrow \mathbb{S}^{2}$ equal to the inverse stereographic projection. Then $f_{t}$ converges smoothly on compact subsets of $\mathbb{C} \cup\{\infty\} \backslash\left\{\pi_{1}, \cdots, \pi_{n}\right\}$ to $f_{0}$. Indeed, on compact subsets of $\mathbb{C} \backslash\left\{\pi_{1}, \cdots, \pi_{n}\right\}, \xi_{t}$ converges uniformly to the standard spherical potential $\xi_{0}=\left(\begin{array}{cc}0 & \lambda^{-1} \\ 0 & 0\end{array}\right) d z$, with the same initial data; and in a neighborhood of $\infty, \xi_{t} \cdot G_{\infty}$ converges uniformly to $\xi_{0} \cdot G_{\infty}$. Let $M_{t}=f_{t}\left(\Sigma_{t}\right)$, where $\Sigma_{t}$ is defined by Equation (23).

Proposition 6. If all weights $\tau_{i}$ are positive and the angle between $u_{i}$ and $u_{j}$ is greater than $\frac{\pi}{3}$ for all $j \neq i$, then for $t>0$ small enough, $M_{t}$ is embedded.

Proof: we continue with the notations of Section 10.1. In particular, $\epsilon$ is the number given by our application of Theorem 4 and is fixed.

- For $i \in[1, n]$, we denote $\mathcal{D}_{i, t}$ the Delaunay surface $f_{i, t}^{\mathcal{D}}\left(\mathbb{C}^{*}\right)$. By Point 3 of Theorem 4 for $t$ small enough and for all $r \leq \epsilon$, the projection from $f_{t}\left(D^{*}\left(p_{i, t}, r\right)\right)$ to $\mathcal{D}_{i, t}$ is a diffeomorphism onto its image which we denote $U_{i, t, r}$. In other words, $f_{t}\left(D^{*}\left(p_{i, t}, r\right)\right)$ is the normal graph on $U_{i, t, r} \subset \mathcal{D}_{i, t}$ of a function which, by Point 1 of Theorem 4 , is bounded by $c_{1} t$ where $c_{1}=c \epsilon^{\alpha}$. We denote $V_{i, t, r}=\operatorname{Tub}_{c_{1} t} U_{i, t, r}$ the tubular neighborhood of radius $c_{1} t$ of $U_{i, t, r}$. We have $f_{t}\left(D^{*}\left(p_{i, t}, r\right)\right) \subset$ $V_{i, t, r}$ (see Figure 2).

- For $r>0$, let

$$
\Omega_{t, r}=\left\{z \in \mathbb{C}: \forall i \in[1, n],\left|z-p_{i, t}\right|>r\right\} \cup\{\infty\} .
$$

For $t>0$ small enough, $f_{t}\left(\Omega_{t, \frac{\epsilon}{8}}\right)$ is the normal graph, on a domain of the sphere $\mathbb{S}^{2}$, of a function which is bounded by $c_{2} t$ for some constant $c_{2}$. For $r \in\left[\frac{\epsilon}{8}, \epsilon\right]$, we denote $U_{t, r}$ the projection of $f_{t}\left(\Omega_{t, r}\right)$ on the sphere and $V_{t, r}=\operatorname{Tub}_{c_{2} t} U_{t, r}$ the tubular neighborhood of radius $c_{2} t$ of $U_{t, r}$. We have $f_{t}\left(\Omega_{t, r}\right) \subset V_{t, r}$.

For $p \in \mathbb{R}^{3}$, we denote $\mathbb{S}^{2}(p)$ the unit sphere centered at $p$.

Claim 4. For $r \in\left[\frac{\epsilon}{8}, \epsilon\right]$ :

$$
\lim _{t \rightarrow 0} \bar{V}_{t, r}=\mathbb{S}^{2}(0) \backslash \bigcup_{i=1}^{n} f_{0}\left(D\left(\pi_{i}, r\right)\right)=: L_{r}
$$




$$
\forall i \in[1, n], \quad \lim _{t \rightarrow 0} \bar{V}_{i, t, r}=f_{0}\left(\bar{D}\left(\pi_{i}, r\right)\right) \cup \bigcup_{k \geq 1} \mathbb{S}^{2}\left(2 k u_{i}\right)=: L_{i, r} .
$$

In Equation (30), the limit is for the Hausdorff distance $d_{\mathcal{H}}$, and in Equation (31) for the Hausdorff distance on compact subsets of $\mathbb{R}^{3}: \lim _{t \rightarrow 0} A_{t}=L$ means that for all compact $K \subset \mathbb{R}^{3}, \lim _{t \rightarrow 0} d_{\mathcal{H}}\left(A_{t} \cap\right.$ $K, L \cap K)=0$.

Proof:

1. The Hausdorff distance between $\bar{V}_{t, r}$ and $f_{t}\left(\bar{\Omega}_{t, r}\right)$ is at most $2 c_{2} t$, so

$$
\lim _{t \rightarrow 0} \bar{V}_{t, r}=\lim _{t \rightarrow 0} f_{t}\left(\bar{\Omega}_{t, r}\right)=f_{0}\left(\mathbb{C} \cup\{\infty\} \backslash \bigcup_{i=1}^{n} D\left(\pi_{i}, r\right)\right)=L_{r} .
$$

2. It is known that as $t \rightarrow 0$, the family of Delaunay surfaces $\mathcal{D}_{i, t}$ converges, up to rigid motions, to an infinite chain of unit-spheres. Since the axis of $\mathcal{D}_{i, t}$ converges as $t \rightarrow 0$ to the line spanned by $u_{i}$, the family $\mathcal{D}_{i, t}$ converges, up to translation along this line, to a chain of unit-spheres with centers on this line. We have

$$
\lim _{t \rightarrow 0} f_{i, t}^{\mathcal{D}}\left(\bar{D}(0, r) \backslash D\left(0, \frac{r}{2}\right)\right)=\lim _{t \rightarrow 0} f_{t}\left(\bar{D}\left(p_{i, t}, r\right) \backslash D\left(p_{i, t}, \frac{r}{2}\right)\right)=f_{0}\left(\bar{D}\left(\pi_{i}, r\right) \backslash D\left(\pi_{i}, \frac{r}{2}\right)\right) \subset \mathbb{S}^{2}(0) .
$$

Hence $\mathcal{D}_{i, t}$ converges to the union of the spheres $\mathbb{S}^{2}\left(2 k u_{i}\right)$ for $k \in \mathbb{Z}$ and

$$
\lim _{t \rightarrow 0} \bar{V}_{i, t, r}=\lim _{t \rightarrow 0} \overline{f_{t}\left(D^{*}\left(p_{i, t}, r\right)\right)}=\lim _{t \rightarrow 0} \overline{f_{i, t}^{\mathcal{D}}\left(D^{*}(0, r)\right)}=f_{0}\left(\bar{D}\left(\pi_{i}, r\right)\right) \cup \bigcup_{k \geq 1} \mathbb{S}^{2}\left(2 k u_{i}\right)=L_{i, r} .
$$

Claim 5. There exists a positive $T^{\prime}<T$ such that for $0<t<T^{\prime}$ and $r \in\left[\frac{\epsilon}{4}, \epsilon\right]$ :

$$
\begin{gathered}
\forall j \neq i, \quad \bar{V}_{i, t, r} \cap \bar{V}_{j, t, r}=\emptyset \\
\forall i, \quad \bar{V}_{i, t, \frac{r}{2}} \cap \bar{V}_{t, r}=\emptyset
\end{gathered}
$$

Proof: The angle hypothesis of Proposition 6 ensures that the spheres $\mathbb{S}^{2}\left(2 k u_{i}\right)$ and $\mathbb{S}^{2}\left(2 \ell u_{j}\right)$ for $j \neq i$ and $k, \ell \geq 1$ do not intersect. Hence the limit sets $L_{i, r}$ for $i \in[1, n]$ are disjoint. Let $\delta>0$ be the smallest distance between $L_{i, r}$ and $L_{j, r}$ for $j \neq i$. Let $K=\bar{B}(0,4)$. There exists a positive $T^{\prime} \leq T$ such that for $t<T^{\prime}$ and $i \in[1, n]$,

$$
d_{\mathcal{H}}\left(\bar{V}_{i, t, r} \cap K, L_{i, r} \cap K\right)<\frac{\delta}{2}
$$

Hence for $t<T^{\prime}$ and $j \neq i, \bar{V}_{i, t, r}$ and $\bar{V}_{j, t, r}$ do not intersect inside $K$. Since it is clear that they don't intersect outside $K$, Equation 32 follows. Equation 33 is proved in the same way, observing that the limit sets $L_{i, \frac{r}{2}}$ and $L_{r}$ are disjoint.

Claim 6. For $0<t<T^{\prime}$ and $i \in[1, n]$ :

$$
\begin{gathered}
f_{t}^{-1}\left(V_{i, t, \frac{\epsilon}{2}}\right)=D^{*}\left(p_{i, t}, \frac{\epsilon}{2}\right) \\
f_{t}^{-1}\left(V_{t, \frac{\epsilon}{4}}\right)=\Omega_{t, \frac{\epsilon}{4}}
\end{gathered}
$$

Proof:

1. Let $z \in f_{t}^{-1}\left(V_{i, t, \frac{\epsilon}{2}}\right)$. By Equation (33) with $r=\epsilon, f_{t}(z) \notin \bar{V}_{t, \epsilon}$ hence $z \notin \bar{\Omega}_{t, \epsilon}$. So there exists $j \in[1, n]$ such that $z \in D^{*}\left(p_{j, t}, \epsilon\right)$. Equation (32) with $r=\epsilon$ yields $j=i$. Since the projection from $f_{i, t}\left(D^{*}\left(p_{i, t}, \epsilon\right)\right)$ to $U_{i, t, \epsilon}$ is a diffeomorphism, $z \in D^{*}\left(p_{i, t}, \frac{\epsilon}{2}\right)$.

2. Let $z \in f_{t}^{-1}\left(V_{t, \frac{\epsilon}{4}}\right)$. Equation (33) with $r=\frac{\epsilon}{4}$ implies that for all $i \in[1, n], f_{t}(z) \notin \bar{V}_{i, t, \frac{\epsilon}{8}}$ so $z \notin \bar{D}\left(p_{i, t}, \frac{\epsilon}{8}\right)$. Hence $z \in \Omega_{t, \frac{\epsilon}{8}}$. Since the projection from $f_{t}\left(\Omega_{t, \frac{\epsilon}{8}}\right)$ to $U_{t, \frac{\epsilon}{8}}$ is a diffeomorphism, $z \in \Omega_{t, \frac{\epsilon}{4}}$. 
Claim 6 implies that $M_{t}$ is embedded. Indeed, the open sets $V_{i, t, \frac{\epsilon}{2}}$ for $i \in[1, n]$ and $V_{t, \frac{\epsilon}{4}}$ cover $M_{t}$ and the intersection of $M_{t}$ with each of these sets is a submanifold, so $M_{t}$ is a submanifold of $\mathbb{R}^{3}$.

10.3. Alexandrov-embeddedness. We recall from [8] the definition of Alexandrov-embeddedness in the non-compact case:

Definition 2. A CMC surface $M$ of finite topology is Alexandrov-embedded if $M$ is properly immersed, if each end of $M$ is embedded, and if there exists a compact 3-manifold $W$ with boundary $\partial W=\Sigma$ and a proper immersion $F: W \backslash\left\{q_{1}, \cdots, q_{n}\right\} \rightarrow \mathbb{R}^{3}$ whose boundary restriction $f: \Sigma \backslash\left\{q_{1}, \cdots, q_{n}\right\} \rightarrow \mathbb{R}^{3}$ parametrizes $M$. Moreover, we require that the mean-curvature normal of $M$ points into $W$.

Proposition 7. If all weights $\tau_{i}$ are positive then for $t>0$ small enough, $M_{t}$ is Alexandrov-embedded.

Proof: the idea is to construct an abstract flat 3-manifold $N_{t}$ in which the $n$ half Delaunay surfaces do not intersect, so $M_{t}$ lifts to an embedded surface in $N_{t}$.

We continue with the notations of Section 10.2 . Let $\mathcal{C}_{i, t}$ be the solid cylinder in $\mathbb{R}^{3}$ bounded by the Delaunay surface $\mathcal{D}_{i, t}$. For positive $\delta$, let

$$
Q_{i, t, \delta}=\left\{x \in \mathbb{R}^{3}: d\left(x, \mathcal{C}_{i, t}\right)<c_{1} t \text { and }\left\langle x, u_{i}\right\rangle>1-\delta\right\} .
$$

Then

$$
\lim _{t \rightarrow 0} \bar{Q}_{i, t, \delta}=\left\{x \in \bar{B}(0,1):\left\langle x, u_{i}\right\rangle \geq 1-\delta\right\} \cup \bigcup_{k \geq 1} \bar{B}\left(2 k u_{i}, 1\right)=: L_{i, \delta}
$$

We have

$$
\lim _{\delta \rightarrow 0} L_{i, \delta} \cap \bar{B}(0,1+\delta)=\left\{u_{i}\right\}
$$

so we may choose $\delta>0$ small enough such that the sets $L_{i, \delta} \cap \bar{B}(0,1+\delta)$ for $i \in[1, n]$ are disjoint. Then for $t$ small enough and $j \neq i$,

$$
Q_{j, t, \delta} \cap Q_{i, t, \delta} \cap B(0,1+\delta)=\emptyset .
$$

Let $N_{t}$ be the flat 3-manifold obtained as the disjoint union of $B(0,1+\delta)$ and $Q_{i, t, \delta}$ for $i \in[1, n]$, gluing $B\left(0,1+\delta\right.$ ) with $Q_{i, t, \delta}$ (with the identity map) where they intersect in $\mathbb{R}^{3}$. Let $\psi_{t}: N_{t} \rightarrow \mathbb{R}^{3}$ be the canonical projection. Then $\psi_{t}$ is an immersion and $N_{t}$ is called an immersed domain. The point here is that the domains $Q_{i, t, \delta}$ for $i \in[1, n]$ are disjoint in $N_{t}$, even if they may intersect in $\mathbb{R}^{3}$.

We have for $t$ small enough

$$
f_{t}\left(\Omega_{t, \frac{\epsilon}{2}}\right) \subset B(0,1+\delta)
$$

and taking $\epsilon$ smaller if necessary,

$$
f_{t}\left(D^{*}\left(p_{i, t}, \epsilon\right)\right) \subset Q_{i, t, \delta} .
$$

Hence we may lift the immersion $f_{t}$ to $\tilde{f}_{t}: \Sigma_{t} \rightarrow N_{t}$ so that $\psi_{t} \circ \tilde{f}_{t}=f_{t}$. Let $\widetilde{M}_{t}=\tilde{f}_{t}\left(\Sigma_{t}\right)$. Since the domains $Q_{i, t, \delta}$ for $i \in[1, n]$ are disjoint in $N_{t}$, the proof of Proposition 6 gives that $\widetilde{M}_{t}$ is embedded in $N_{t}$. Let $W_{t}$ be the domain in $N_{t}$ bounded by $\widetilde{M}_{t}$. Since the Delaunay surfaces are Alexandrov-embedded (in the positive weight case), we may compactify $W_{t}$ by adding one point at infinity per Delaunay end of $\widetilde{M}_{t}$. We take $F_{t}$ to be the restriction of $\psi_{t}$ to $W_{t}$. This proves that $M_{t}$ is Alexandrov embedded. 
10.4. Hopf differential and umbilics. Umbilics are points where the two principal curvatures are equal. On a CMC-1 surface, they are the zeros of the Hopf quadratic differential. Writing the potential $\xi$ as in Equation (1), the Hopf differential is given by

$$
Q(z)=-2 \beta(z, 0) \gamma(z, 0) .
$$

In our case, the Hopf differential is equal to $-2 t \omega_{t}(z, 0) d z$. Since $\omega_{t}$ has a double zero at $\infty$, the Hopf differential is holomorphic at $\infty$ and has $n$ double poles at the punctures. Being a quadratic differential on the Riemann sphere, it has $2 n-4$ zeros, so there are $2 n-4$ umbilics (counting multiplicity). The umbilics converge as $t \rightarrow 0$ to the zeros of $\omega_{0} d z$, which, by Equation (21), is given by

$$
\omega_{0} d z=\sum_{i=1}^{n}\left(\frac{\tau_{i}}{\left(z-\pi_{i}\right)^{2}}-\frac{2 \tau_{i} \overline{\pi_{i}}}{\left(1+\left|\pi_{i}\right|^{2}\right)\left(z-\pi_{i}\right)}\right) d z^{2}
$$

So the limit position of the umbilics can in principle be computed by solving polynomial equations.

\section{APPENDIX A. DERIVATIVE OF THE MONODROMY}

The following proposition is adapted from Proposition 9 in [24].

Proposition 8. Let $\xi_{t}$ be a $C^{1}$ family of matrix-valued 1-forms on a Riemann surface $\Sigma$. Let $\widetilde{\Sigma}$ be the universal cover of $\Sigma$. Fix a point $z_{0}$ in $\Sigma$ and let $\widetilde{z}_{0}$ be a lift of $z_{0}$ to $\widetilde{\Sigma}$. Let $\Phi_{t}$ be a family of solutions of $d \Phi_{t}=\Phi_{t} \xi_{t}$ on $\tilde{\Sigma}$, such that $\Phi_{t}\left(\widetilde{z}_{0}\right)$ does not depend on $t$. Let $\gamma \in \pi_{1}\left(\Sigma, z_{0}\right)$ and let $M(t)$ be the monodromy of $\Phi_{t}$ with respect to $\gamma$. Let $\widetilde{\gamma}$ be the lift of $\gamma$ to $\widetilde{\Sigma}$ such that $\widetilde{\gamma}(0)=\widetilde{z}_{0}$. Then for all $t$,

$$
M^{\prime}(t)=\int_{\widetilde{\gamma}} \Phi_{t} \frac{\partial \xi_{t}}{\partial t} \Phi_{t}^{-1} \times M(t)
$$

Proof: since $\Phi_{t}\left(\widetilde{z}_{0}\right)$ is constant and $\xi_{t}$ depends $C^{1}$ on $t, \Phi_{t}$ depends $C^{1}$ on $t$. Let $\Psi_{t}=\frac{\partial \Phi_{t}}{\partial t}$. By differentiation of the Cauchy Problem satisfied by $\Phi_{t}$ with respect to $t$, we obtain that $\Psi_{t}$ satisfies the following Cauchy Problem on $\widetilde{\Sigma}$ :

$$
\left\{\begin{array}{l}
d \Psi_{t}=\Psi_{t} \xi_{t}+\Phi_{t} \frac{\partial \xi_{t}}{\partial t} \\
\Psi_{t}\left(\widetilde{z}_{0}\right)=0
\end{array}\right.
$$

Following the method of variation of constants, the function $U_{t}=\Psi_{t} \Phi_{t}^{-1}$ satisfies:

$$
\left\{\begin{array}{l}
d U_{t}=\Phi_{t} \frac{\partial \xi_{t}}{\partial t} \Phi_{t}^{-1} \\
U_{t}\left(\widetilde{z}_{0}\right)=0
\end{array}\right.
$$

Hence (writing $\widetilde{\gamma}(1)$ for the endpoint of $\widetilde{\gamma}$ )

$$
U_{t}(\widetilde{\gamma}(1))=\int_{\widetilde{\gamma}} \Phi_{t} \frac{\partial \xi_{t}}{\partial t} \Phi_{t}^{-1}
$$

We have by definition

$$
M(t)=\Phi_{t}(\widetilde{\gamma}(1)) \Phi_{t}\left(\widetilde{z}_{0}\right)^{-1} .
$$

Hence since $\Phi_{t}\left(\widetilde{z}_{0}\right)$ is constant:

$$
\begin{aligned}
M^{\prime}(t) & =\Psi_{t}(\widetilde{\gamma}(1)) \Phi_{t}\left(\widetilde{z}_{0}\right)^{-1} \\
& =U_{t}(\widetilde{\gamma}(1)) \Phi_{t}(\widetilde{\gamma}(1)) \Phi_{t}\left(\widetilde{z}_{0}\right)^{-1} \\
& =\int_{\gamma} \Phi_{t} \frac{\partial \xi_{t}}{\partial t} \Phi_{t}^{-1} M(t) .
\end{aligned}
$$




\section{Appendix B. Smoothness of maps Between Banach SPaCes}

The following proposition is useful to prove that the maps considered in this paper are smooth maps between Banach spaces. The Banach algebra $\mathcal{W}$ is defined in Section 4 . For $R>1$, we denote $\mathbb{A}_{R}$ the annulus $\frac{1}{R}<|\lambda|<R$ in $\mathbb{C}$. For $\mathbf{a}=\left(a_{1}, \cdots, a_{n}\right) \in \mathbb{C}^{n}$ and $\mathbf{r}=\left(r_{1}, \cdots, r_{n}\right) \in(0, \infty)^{n}$, we denote $D(\mathbf{a}, \mathbf{r})$ the polydisk $\prod_{i=1}^{n} D\left(a_{i}, r_{i}\right)$ in $\mathbb{C}^{n}$.

Proposition 9. Let $R>\rho$ and $f: \mathbb{A}_{R} \times D(\mathbf{a}, \mathbf{r}) \rightarrow \mathbb{C}$ be a holomorphic function of $(n+1)$ variables $\left(\lambda, z_{1}, \cdots, z_{n}\right)$. Let

$$
B(\mathbf{a}, \mathbf{r})=\left\{\left(u_{1}, \cdots, u_{n}\right) \in \mathcal{W}^{n}: \forall i \in[1, n],\left\|u_{i}-a_{i}\right\|<r_{i}\right\}
$$

where we identify $a_{i}$ with a constant function in $\mathcal{W}$. Define for $\left(u_{1}, \cdots, u_{n}\right) \in B(\mathbf{a}, \mathbf{r})$ :

$$
F\left(u_{1}, \cdots, u_{n}\right)(\lambda)=f\left(\lambda, u_{1}(\lambda), \cdots, u_{n}(\lambda)\right) .
$$

Then $F: B(\mathbf{a}, \mathbf{r}) \subset \mathcal{W}^{n} \rightarrow \mathcal{W}$ is of class $C^{\infty}$.

Proof: we expand $f$ in Laurent series with respect to $\lambda$ and power series with respect to $z_{1}, \cdots, z_{n}$ :

$$
f\left(\lambda, z_{1}, \cdots, z_{n}\right)=\sum_{k \in \mathbb{Z}} \sum_{i_{1}, \cdots, i_{n}} c_{k i_{1} \cdots i_{n}} \lambda^{k}\left(z_{1}-a_{1}\right)^{i_{1}} \cdots\left(z_{n}-a_{n}\right)^{i_{n}}
$$

For any $\mathbf{r}^{\prime}<\mathbf{r}$ (in the sense $r_{i}^{\prime}<r_{i}$ for all $\left.i\right)$, the series $f\left(\rho^{ \pm 1}, a_{1}+r_{1}^{\prime}, \cdots, a_{n}+r_{n}^{\prime}\right)$ converges absolutely so

$$
\sum_{k \in \mathbb{Z}} \sum_{i_{1}, \cdots, i_{n}}\left|c_{k i_{1} \cdots i_{n}}\right| \rho^{|k|}\left(r_{1}^{\prime}\right)^{i_{1}} \cdots\left(r_{n}^{\prime}\right)^{i_{n}}<\infty .
$$

Let $v \in \mathcal{W}$ be the function defined by $v(\lambda)=\lambda$. Then formally:

$$
F\left(u_{1}, \cdots, u_{n}\right)=\sum_{k \in \mathbb{Z}} \sum_{i_{1}, \cdots, i_{n}} c_{k i_{1} \cdots i_{n}} v^{k}\left(u_{1}-a_{1}\right)^{i_{1}} \cdots\left(u_{n}-a_{n}\right)^{i_{n}} .
$$

Since $\left\|v^{k}\right\|=\rho^{|k|}$ and $\mathcal{W}$ is a Banach algebra, for $\left(u_{1}, \cdots, u_{n}\right) \in B\left(\mathbf{a}, \mathbf{r}^{\prime}\right)$, we have

$$
\left\|c_{k i_{1} \cdots i_{n}} v^{k}\left(u_{1}-a_{1}\right)^{i_{1}} \cdots\left(u_{n}-a_{n}\right)^{i_{n}}\right\| \leq\left|c_{k i_{1} \cdots i_{n}}\right| \rho^{|k|}\left(r_{1}^{\prime}\right)^{i_{1}} \cdots\left(r_{n}^{\prime}\right)^{i_{n}}
$$

Hence by Inequality (34), the series (35) converges normally, so $F\left(u_{1}, \cdots, u_{n}\right) \in \mathcal{W}$ and $F$ is of class $C^{\infty}$ on $B\left(\mathbf{a}, \mathbf{r}^{\prime}\right)$. (See Theorem 11.12 in [1] for the smoothness of maps defined by power series in Banach spaces.)

\section{REFERENCES}

[1] S. B. Chae. Holomorphy and calculus in normed spaces. Monographs and textbooks in pure and applied mathematics, vol. 92 (1985).

[2] J. Dorfmeister, G. Haak: Constant mean curvature surfaces with periodic metric. Pacific Journal of Mathematics 182 (1998), 229-287.

[3] J. Dorfmeister, F. Pedit, H. Wu: Weierstrass type representation of harmonic maps into symmetric spaces. Communications in Analysis and Geometry 6 (1998), 633-668.

[4] J. Dorfmeister, H. Wu: Construction of constant mean curvature n-noids from holomorphic potentials. Mathematische Zeitschrift 258 (2008), 773-803.

[5] O. Forster: Lectures on Riemann surfaces. Graduate texts in Mathematics, Springer Verlag.

[6] S. Fujimori, S. Kobayashi, W. Rossman: Loop group methods for constant mean curvature surfaces. arXiv:math/0602570

[7] A. Gerding, F. Pedit, N. Schmitt: Constant mean curvature surfaces: an integrable systems perspective. Harmonic maps and differential geometry, Contemp. Math. 542 (2011), Amer. Math. Soc., 7-39.

[8] K. Große-Brauckmann, R. Kusner, J. Sullivan: Triunduloids: embedded constant mean curvature surfaces with three ends and genus zero. J. Reine Angew. Math. 564 (2003), 35-61.

[9] K. Große-Brauckmann, R. Kusner, J. Sullivan: Coplanar constant mean curvature surfaces. Comm. Anal. Geom. 15 (2007), no. 5, 985-1023.

[10] L. Heller, S. Heller, N. Schmitt: Navigating the Space of Symmetric CMC Surfaces. arXiv:1501.01929.

[11] S. Heller: Higher genus minimal surfaces in $\mathbb{S}^{3}$ and stable bundles. J. Reine Angew. Math. 685 (2013), $105-122$. 
[12] S. Heller: Lawson's genus two surface and meromorphic connections. Mathematische Zeitschrift 274 (2013), 745-760.

[13] S. Heller: A spectral curve approach to Lawson symmetric CMC surfaces of genus 2. Math. Annalen 360, Issue 3 (2014), 607-652.

[14] N. Kapouleas: Complete constant mean curvature surfaces in euclidean three-space. Annals of Mathematics 131 (1990), 239-330.

[15] M. Kilian, S. Kobayashi, W. Rossman, N. Schmitt: Constant mean curvature surfaces of any positive genus. J. London Math. Soc. 72 (2005), 258-272.

[16] M. Kilian, I. McIntosh, N. Schmitt: New constant mean curvature surfaces. Experiment. Math. 9 (2000), 595-611.

[17] M. Kilian, W. Rossman, N. Schmitt: Delaunay ends of constant mean curvature surfaces. Compositio Mathematica 144 (2008), 186-220.

[18] N. Korevaar, R. Kusner, B. Solomon: The structure of complete embedded surfaces with constant mean curvature. J. Diff. Geom. 30 (1989), 465-503.

[19] R. Kusner, R. Mazzeo, D. Pollack: The moduli space of complete embedded constant mean curvature surfaces. GAFA 6, Issue 1 (1996), 120-137.

[20] T. Raujouan: On Delaunay ends in the DPW method. arXiv:1710.00768

[21] N. Schmitt: Constant mean curvature n-noids with platonic symmetries. arXiv:math/0702469.

[22] N. Schmitt, M. Kilian, S. Kobayashi, W. Rossman: Unitarization of monodromy representations and constant mean curvature trinoids in 3-dimensional space forms. Journal of the London Mathematical Society 75 (2007), 563-581.

[23] M. Taylor: Introduction to Differential Equations. Pure and Applied Undergraduate Texts 14, American Math. Soc. (2011).

[24] M. Traizet: Opening nodes on horosphere packings. Trans. Amer. Math. Soc. 368 (2016), 5701-5725.

Martin Traizet

Institut Denis Poisson

Université de Tours, 37200 Tours, France

martin.traizet@univ-tours.fr 